Bull. Soc. math. France

131 (4), 2003, p. 527-551

\title{
TRANSFORMATION DE FOURIER HOMOGÈNE
}

\author{
PAR GÉRARD LAUMON
}

RÉSumé. - Dans leur démonstration de la correspondance de Drinfeld-Langlands, Frenkel, Gaitsgory et Vilonen utilisent la transformation de Fourier géométrique, ce qui les oblige à travailler soit avec les faisceaux $\ell$-adiques en caractéristique $p>0$, soit avec les $\mathcal{D}$-Modules en caractéristique 0. En fait, ils n'utilisent cette transformation de Fourier géométrique que pour des faisceaux homogènes pour lesquels on s'attend à avoir une transformation de Fourier sur $\mathbb{Z}$. L'objet de cette note est de proposer une telle transformation de Fourier qui prolonge la transformation de Radon géométrique étudiée par Brylinski.

Abstract (Homogeneous Fourier transformation). - In their proof of the DrinfeldLanglands correspondence, Frenkel, Gaitsgory and Vilonen make use of a geometric Fourier transformation. Therefore, they work either with $\ell$-adic sheaves in characteristic $p>0$, or with $\mathcal{D}$-modules in characteristic 0 . Actually, they only need to consider the Fourier transforms of homogeneous sheaves for which one expects a geometric Fourier transformation over $\mathbb{Z}$. In this note, we propose such a homogeneous geometric Fourier transformation. It extends the geometric Radon transformation which has been studied by Brylinski.

Dans leur démonstration de la correspondance de Drinfeld-Langlands [5], Frenkel, Gaitsgory et Vilonen utilisent la transformation de Fourier géométrique, ce qui les obligent soit à supposer que le corps de base est de caractéristique $p>0$ et à travailler avec les faisceaux $\ell$-adiques, soit à supposer qu'il est

Texte reçu le 8 novembre 2002, accepté le 17 janvier 2003

Gérard Laumon, Université Paris-Sud et CNRS, UMR 8628, Mathématique, Bât. 425, F-91405 Orsay Cedex (France) • E-mail : Gerard.Laumon@math.u-psud.fr

Classification mathématique par sujets (2000). — 11T23, 14F20, 14F22.

Mots clefs. - Transformation de Fourier, faisceaux pervers, champs algébriques.

BULLETIN DE LA SOCIÉTÉ MATHÉMATIQUe DE FRANCE

(C) Société Mathématique de France

$0037-9484 / 2003 / 527 / \$ 5.00$ 
de caractéristique 0 et à travailler avec les $\mathcal{D}$-Modules. En fait, ils n'utilisent cette transformation de Fourier géométrique que pour des faisceaux homogènes pour lesquels on s'attend à avoir une transformation de Fourier sur $\mathbb{Z}$ qui prolonge la transformation de Radon géométrique étudiée par Brylinski dans [3]. L'objet de cette note est de proposer une telle transformation de Fourier.

Je remercie Bourbaki de m'avoir donné l'occasion de réfléchir à ce problème en préparant un exposé à son séminaire [10]. Je remercie aussi L. Illusie pour son aide durant la rédaction de cette note. Je remercie enfin S. Lysenko et le rapporteur pour leurs commentaires et suggestions.

\section{La transformation de Fourier homogène}

1.1. Soient $S$ un schéma et $\pi: V \rightarrow S$ un fibré vectoriel de rang constant $r$ sur $S$. Le $S$-schéma en groupes multiplicatif $\mathbb{G}_{\mathrm{m}, S}$ agit par homothétie sur $V$ et on peut former le $S$-champ quotient

$$
V \stackrel{\rho}{\longrightarrow} V=\left[V / \mathbb{G}_{\mathrm{m}, S}\right] \stackrel{\bar{\pi}}{\longrightarrow} S
$$

de $V$ par cette action. Rappelons que, pour tout $S$-schéma $U$, un objet de la catégorie $\mathcal{V}(U)$ est un couple $(\mathcal{L}, x)$ formé d'un fibré en droites $\mathcal{L}$ sur $U$ et d'un morphisme de $\mathcal{O}_{U}$-Modules $x: \mathcal{L} \rightarrow \mathcal{O}_{U} \otimes_{\mathcal{O}_{S}} V$ où $V$ est ici considéré comme un $\mathcal{O}_{S}$-Module localement libre de rang constant $r$. Le $S$-champ $\mathcal{V}$ est algébrique, de type fini et lisse purement de dimension relative $r-1$, et le morphisme quotient $\rho$ est un $\mathbb{G}_{\mathrm{m}, S}$-torseur représentable. Pour $V=\mathbb{A}_{S}^{1}$ le fibré trivial de rang 1 , on note $\mathcal{A}_{S}$ le champ $\mathcal{V}$ correspondant.

Soient $\pi^{\vee}: V^{\vee} \rightarrow S$ le fibré vectoriel dual de $\pi$ et

$$
\langle,\rangle: V^{\vee} \times_{S} V \longrightarrow \mathbb{A}_{S}^{1}
$$

l'accouplement canonique. Comme précédemment, on forme le $S$-champ quotient

$$
V^{\vee} \stackrel{\rho^{\vee}}{\longrightarrow} V^{\vee}=\left[V^{\vee} / \mathbb{G}_{\mathrm{m}, S}\right] \stackrel{\bar{\pi}^{\vee}}{\longrightarrow} S
$$

et l'accouplement $\langle$,$\rangle induit un S$-morphisme

$$
\mu: \mathcal{V}^{\vee} \times_{S} \mathcal{V} \longrightarrow \mathcal{A}_{S}
$$

Plus précisément, si $U$ est un $S$-schéma, $\mu$ envoie un objet $((\mathcal{M}, y),(\mathcal{L}, x))$ de $\left(\mathcal{V}^{\vee} \times{ }_{S} \mathcal{V}\right)(U)$ sur l'objet

$$
\left(\mathcal{M} \otimes_{\mathcal{O}_{U}} \mathcal{L},\langle y, x\rangle: \mathcal{M} \otimes_{\mathcal{O}_{U}} \mathcal{L} \rightarrow \mathcal{O}_{U} \otimes_{\mathcal{O}_{S}} V^{\vee} \otimes_{\mathcal{O}_{S}} V \rightarrow \mathcal{O}_{U}\right)
$$

de $\mathcal{A}_{S}(U)$. 
1.2. On fixe un nombre premier $\ell$ inversible sur $S$ et une clôture algébrique $\overline{\mathbb{Q}}_{\ell}$ de $\mathbb{Q}_{\ell}$. On suppose que $S$ est de type fini sur un anneau régulier $R$ de dimension $\leq 1$, de sorte que l'on devrait disposer d'un formalisme $\ell$-adique des six opérations de Grothendieck sur la catégorie des $S$-champs algébriques de type fini (cf. [4], [1] et [11]), ce que nous admettrons ici. Pour tout $S$-champ $X$ de type fini, on a donc des catégories dérivées $D_{\mathrm{c}}^{\mathrm{b}}\left(\mathcal{X}, \overline{\mathbb{Q}}_{\ell}\right) \subset D_{\mathrm{c}}^{ \pm}\left(\mathcal{X}, \overline{\mathbb{Q}}_{\ell}\right)$, avec

- des foncteurs

$$
\begin{array}{ll}
f_{*}: D_{\mathrm{c}}^{+}\left(\mathcal{X}, \overline{\mathbb{Q}}_{\ell}\right) \rightarrow D_{\mathrm{c}}^{+}\left(\mathrm{y}, \overline{\mathbb{Q}}_{\ell}\right), & f_{!}: D_{\mathrm{c}}^{-}\left(\mathcal{X}, \overline{\mathbb{Q}}_{\ell}\right) \rightarrow D_{\mathrm{c}}^{-}\left(\mathrm{y}, \overline{\mathbb{Q}}_{\ell}\right), \\
f^{*}: D_{\mathrm{c}}^{ \pm, \mathrm{b}}\left(\mathrm{y}, \overline{\mathbb{Q}}_{\ell}\right) \rightarrow D_{\mathrm{c}}^{ \pm, \mathrm{b}}\left(\mathcal{X}, \overline{\mathbb{Q}}_{\ell}\right), & f^{!}: D_{\mathrm{c}}^{ \pm, \mathrm{b}}\left(\mathrm{y}, \overline{\mathbb{Q}}_{\ell}\right) \rightarrow D_{\mathrm{c}}^{ \pm, \mathrm{b}}\left(\mathcal{X}, \overline{\mathbb{Q}}_{\ell}\right),
\end{array}
$$

pour tout $S$-morphisme $f: \mathcal{X} \rightarrow \mathcal{Y}$ de $S$-champs algébriques de type fini, les foncteurs $f_{*}$ et $f$ ! respectant $D_{\mathrm{c}}^{\mathrm{b}}$ lorsque $f$ est représentable;

- un foncteur «Hom interne »

$$
\mathcal{H} \text { om: } D_{\mathrm{c}}^{\mathrm{b}}\left(\mathcal{X}, \overline{\mathbb{Q}}_{\ell}\right)^{\mathrm{opp}} \times D_{\mathrm{c}}^{ \pm, \mathrm{b}}\left(\mathcal{X}, \overline{\mathbb{Q}}_{\ell}\right) \longrightarrow D_{\mathrm{c}}^{ \pm, \mathrm{b}}\left(\mathcal{X}, \overline{\mathbb{Q}}_{\ell}\right) ;
$$

- un foncteur de dualité involutif

$$
D_{X}: D_{\mathrm{c}}^{ \pm, \mathrm{b}}\left(\mathcal{X}, \overline{\mathbb{Q}}_{\ell}\right)^{\mathrm{opp}} \longrightarrow D_{\mathrm{c}}^{\mp, \mathrm{b}}\left(\mathcal{X}, \overline{\mathbb{Q}}_{\ell}\right)
$$

défini par

$$
D_{x}(K)=\mathcal{H} \text { om }\left(K, \varepsilon ! \overline{\mathbb{Q}}_{\ell}[2 d](d)\right)
$$

où $\varepsilon: X \rightarrow \operatorname{Spec}(R)$ est le morphisme structural et $d(=0$ ou 1$)$ est la dimension de $R$;

- des isomorphismes canoniques de foncteurs

$$
D_{\mathcal{X}} \circ f_{*} \cong f_{!} \circ D_{\mathcal{X}} \text { et } \quad D_{\mathcal{X}} \circ f^{*} \cong f^{!} \circ D_{X} ;
$$

- deux produits tensoriels internes échangés par la dualité,

$$
\begin{aligned}
\otimes: D_{\mathrm{c}}^{ \pm, \mathrm{b}}\left(X, \overline{\mathbb{Q}}_{\ell}\right) \times D_{\mathrm{c}}^{ \pm, \mathrm{b}}\left(X, \overline{\mathbb{Q}}_{\ell}\right) & \longrightarrow D_{\mathrm{c}}^{ \pm, \mathrm{b}}\left(X, \overline{\mathbb{Q}}_{\ell}\right) \\
\widetilde{\otimes}: D_{\mathrm{c}}^{ \pm, \mathrm{b}}\left(X, \overline{\mathbb{Q}}_{\ell}\right) \times D_{\mathrm{c}}^{ \pm, \mathrm{b}}\left(X, \overline{\mathbb{Q}}_{\ell}\right) & \longrightarrow D_{\mathrm{c}}^{ \pm, \mathrm{b}}\left(X, \overline{\mathbb{Q}}_{\ell}\right), \\
\left(K_{1}, K_{2}\right) & \longmapsto D_{X}\left(D_{X}\left(K_{1}\right) \otimes D_{X}\left(K_{2}\right)\right) ;
\end{aligned}
$$

- un isomorphisme canonique $\mathcal{H} \operatorname{Com}(K, L) \cong D_{x}(K) \widetilde{\otimes} L$ fonctoriel en $(K, L)$ dans $D_{\mathrm{c}}^{\mathrm{b}}\left(\mathcal{X}, \overline{\mathbb{Q}}_{\ell}\right)^{\text {opp }} \times D_{\mathrm{c}}^{ \pm, \mathrm{b}}\left(\mathcal{X}, \overline{\mathbb{Q}}_{\ell}\right)$.

On dispose aussi de la sous-catégorie strictement pleine $\operatorname{Perv}\left(X, \overline{\mathbb{Q}}_{\ell}\right) \subset$ $D_{\mathrm{c}}^{\mathrm{b}}\left(X, \overline{\mathbb{Q}}_{\ell}\right)$ des $\overline{\mathbb{Q}}_{\ell}$-faisceaux pervers pour la fonction de dimension rectifiée introduite par M. Artin (cf. [2] et [6]). Si $X$ est un schéma de type fini sur $S$, rappelons que cette fonction est définie par

$$
\delta(x)=\operatorname{dim}(\overline{\{\mathfrak{p}\}})+\text { deg. } \operatorname{tr}(\kappa(x) / \kappa(\mathfrak{p})), \quad \forall x \in X,
$$

où $\mathfrak{p} \in \operatorname{Spec}(R)$ est l'image de $x, \overline{\{\mathfrak{p}\}}$ est l'adhérence de $\{\mathfrak{p}\}$ dans $\operatorname{Spec}(R), \kappa(x)$ est le corps résiduel de $x$ et $\kappa(\mathfrak{p})$ est le corps des fractions de $R / \mathfrak{p}$, et rappelons 
que $K \in$ ob $D_{\mathrm{c}}^{\mathrm{b}}\left(X, \overline{\mathbb{Q}}_{\ell}\right)$ est un $\overline{\mathbb{Q}}_{\ell}$-faisceau pervers si et seulement si, pour tout point $x \in X$, on a

$$
\mathcal{H}^{n} i_{x}^{*} K=(0), \forall n>-\delta(x) \quad \text { et } \quad \mathcal{H}^{n} i_{x}^{!} K=(0), \forall n<-\delta(x),
$$

où $i_{x}:\{x\} \hookrightarrow X$ est l'inclusion.

La sous-catégorie $\operatorname{Perv}\left(X, \overline{\mathbb{Q}}_{\ell}\right) \subset D_{\mathrm{c}}^{\mathrm{b}}\left(X, \overline{\mathbb{Q}}_{\ell}\right)$ est stable par la dualité $D_{X}$ et elle est nœthérienne et artinienne (tous ses objets sont de longueur finie).

1.3. L'inclusion naturelle $\mathbb{G}_{\mathrm{m}, S} \hookrightarrow \mathbb{A}_{S}^{1}$ passe au quotient en une immersion ouverte

$$
\beta: S=\left[\mathbb{G}_{\mathrm{m}, S} / \mathbb{G}_{\mathrm{m}, S}\right] \longleftrightarrow \mathcal{A}_{S} .
$$

On note

$$
\Psi=\beta_{*} \overline{\mathbb{Q}}_{\ell} \in D_{\mathrm{c}}^{\mathrm{b}}\left(\mathcal{A}_{S}, \overline{\mathbb{Q}}_{\ell}\right)
$$

l'image directe totale du faisceau constant $\overline{\mathbb{Q}}_{\ell}$ sur $S$ par cette dernière immersion ouverte. Le théorème de pureté cohomologique nous donne un triangle distingué

$$
\overline{\mathbb{Q}}_{\ell} \longrightarrow \Psi \longrightarrow \alpha_{*} \overline{\mathbb{Q}}_{\ell}[-1](-1) \longrightarrow
$$

dans $D_{\mathrm{c}}^{\mathrm{b}}\left(\mathcal{A}_{S}, \overline{\mathbb{Q}}_{\ell}\right)$, où $\alpha: B\left(\mathbb{G}_{\mathrm{m}, S}\right)=\left[S / \mathbb{G}_{\mathrm{m}, S}\right] \hookrightarrow \mathcal{A}_{S}$ est l'immersion fermée complémentaire de $\beta$.

Lemme 1.4. - (i) Pour toute section $a: S \rightarrow \mathbb{A}_{S}^{1}$ de $\mathbb{A}_{S}^{1}$, on a

$$
h_{!} v_{*} \overline{\mathbb{Q}}_{\ell}=(0)
$$

où $v: \mathbb{A}_{S}^{1}-a(S) \hookrightarrow \mathbb{A}_{S}^{1}$ est l'ouvert complémentaire de l'image de la section et $h: \mathbb{A}_{S}^{1} \rightarrow S$ est le morphisme structural.

(ii) On a un isomorphisme canonique

$$
\alpha^{*} \beta_{*} \overline{\mathbb{Q}}_{\ell} \stackrel{\sim}{\longrightarrow} g ! \overline{\mathbb{Q}}_{\ell}[1]
$$

où $g: S \rightarrow B\left(\mathbb{G}_{\mathrm{m}, S}\right)$ est le $\mathbb{G}_{\mathrm{m}, S}$-torseur universel.

Démonstration. - Commençons par l'assertion (i). Par le théorème de changement de base propre, on peut supposer que $S$ est le spectre d'un corps algébriquement clos, et donc que $a$ est un point fermé de $\mathbb{A}^{1}$ (pour alléger la rédaction, on supprime l'indice $S$ des notations). Il s'agit alors de démontrer que

$$
R \Gamma_{\mathrm{c}}\left(\mathbb{A}^{1}, v_{*} \overline{\mathbb{Q}}_{\ell}\right)=(0),
$$

ou ce qui revient au même par dualité, que

$$
R \Gamma\left(\mathbb{A}^{1}, v ! \overline{\mathbb{Q}}_{\ell}\right)=(0) .
$$

Or, on a le triangle distingué

$$
R \Gamma\left(\mathbb{A}^{1}, v_{!} \overline{\mathbb{Q}}_{\ell}\right) \longrightarrow R \Gamma\left(\mathbb{A}^{1}, \overline{\mathbb{Q}}_{\ell}\right) \longrightarrow \overline{\mathbb{Q}}_{\ell} \longrightarrow
$$

où la deuxième flèche est la flèche de restriction à $\{a\} \subset \mathbb{A}^{1}$ et est donc un isomorphisme, d'où la conclusion. 
Démontrons maintenant l'assertion (ii). Pour cela considérons le triangle distingué

$$
\beta_{!} \overline{\mathbb{Q}}_{\ell} \longrightarrow \beta_{*} \overline{\mathbb{Q}}_{\ell} \longrightarrow \alpha_{*} \alpha^{*} \beta_{*} \overline{\mathbb{Q}}_{\ell}
$$

dans $D_{\mathrm{c}}^{\mathrm{b}}\left(\mathcal{A}_{S}, \overline{\mathbb{Q}}_{\ell}\right)$ et appliquons lui le foncteur $h_{!}$pour le morphisme

$$
h: \mathcal{A}_{S}=\left[\mathbb{A}_{S}^{1} / \mathbb{G}_{\mathrm{m}, S}\right] \longrightarrow\left[S / \mathbb{G}_{\mathrm{m}, S}\right]=B\left(\mathbb{G}_{\mathrm{m}, S}\right)
$$

qui est induit par le morphisme structural $\mathbb{A}_{S}^{1} \rightarrow S$. On obtient le triangle distingué

$$
g ! \overline{\mathbb{Q}}_{\ell} \longrightarrow h_{!} \beta_{*} \overline{\mathbb{Q}}_{\ell} \longrightarrow \alpha^{*} \beta_{*} \overline{\mathbb{Q}}_{\ell}
$$

dans $D_{\mathrm{c}}^{\mathrm{b}}\left(B\left(\mathbb{G}_{\mathrm{m}, S}\right), \overline{\mathbb{Q}}_{\ell}\right)$. Ce dernier triangle dégénère en un isomorphisme

$$
\alpha^{*} \beta_{*} \overline{\mathbb{Q}}_{\ell} \stackrel{\sim}{\longrightarrow} g ! \overline{\mathbb{Q}}_{\ell}[1]
$$

puisqu'on a $h_{!} \beta_{*} \overline{\mathbb{Q}}_{\ell}=(0)$ d'après la partie (i) déjà démontrée.

DÉFINITION 1.5. - La transformation de Fourier homogène est le foncteur

$$
\operatorname{Four}_{\mathcal{V} / S}: D_{\mathrm{c}}^{\mathrm{b}}\left(\mathcal{V}, \overline{\mathbb{Q}}_{\ell}\right) \longrightarrow D_{\mathrm{c}}^{-}\left(\mathcal{V}^{\vee}, \overline{\mathbb{Q}}_{\ell}\right)
$$

défini par

$$
\operatorname{Four}_{\mathcal{V} / S}(K)=\operatorname{pr}_{!}^{\vee}\left(\operatorname{pr}^{*} K \otimes \mu^{*} \Psi\right)[r-1]
$$

où $\mathrm{pr}^{\vee}$ et pr sont les projections canoniques de $\mathcal{V}^{\vee} \times{ }_{S} \mathcal{V}$.

Les immersions ouvertes $V^{\circ} \hookrightarrow V$ et $V^{\vee \circ} \hookrightarrow V^{\vee}$ des complémentaires des sections nulles des fibrés vectoriels $V$ et $V^{\vee}$ passent au quotient en des immersions ouvertes

$$
\begin{gathered}
j: \mathbb{P}(V)=\mathcal{V}^{\circ}=\left[V^{\circ} / \mathbb{G}_{\mathrm{m}, S}\right] \longleftrightarrow \mathcal{V}, \\
j^{\vee}: \mathbb{P}\left(V^{\vee}\right)=\mathcal{V}^{\vee \circ}=\left[V^{\vee \circ} / \mathbb{G}_{\mathrm{m}, S}\right] \longleftrightarrow \mathcal{V}^{\vee}
\end{gathered}
$$

où $\mathbb{P}(V)$ et $\mathbb{P}\left(V^{\vee}\right)$ sont les $S$-fibrés projectifs des droites de $V / S$ et $V^{\vee} / S$ respectivement. Notons

$$
I: H \longleftrightarrow \mathbb{P}\left(V^{\vee}\right) \times{ }_{S} \mathbb{P}(V)
$$

l'hypersurface d'incidence, quotient du fermé de $V^{\vee \circ} \times_{S} V^{\circ}$ d'équation $\langle w, v\rangle=0$. Notons enfin

$$
J:\left(\mathbb{P}\left(V^{\vee}\right) \times_{S} \mathbb{P}(V)\right)-H \longleftrightarrow \mathbb{P}\left(V^{\vee}\right) \times_{S} \mathbb{P}(V)
$$

l'immersion ouverte complémentaire du fermé $H$. Comme $H$ est un diviseur lisse sur $S$ dans un schéma lisse sur $S$, une nouvelle application du théorème de pureté donne un triangle distingué

$$
\overline{\mathbb{Q}}_{\ell}[2 r-2] \longrightarrow J_{*} \overline{\mathbb{Q}}_{\ell}[2 r-2] \longrightarrow I_{*} \overline{\mathbb{Q}}_{\ell}[2 r-3](-1) \longrightarrow
$$

dans $D_{\mathrm{c}}^{\mathrm{b}}\left(\mathbb{P}\left(V^{\vee}\right) \times_{S} \mathbb{P}(V), \overline{\mathbb{Q}}_{\ell}\right)$. 
Proposition 1.6. - Le foncteur composé

$$
\left(j^{\vee}\right)^{*} \circ \operatorname{Four}_{\mathcal{V} / S} \circ j_{!}: D_{\mathrm{c}}^{\mathrm{b}}\left(\mathbb{P}(V), \overline{\mathbb{Q}}_{\ell}\right) \longrightarrow D_{\mathrm{c}}^{-}\left(\mathbb{P}\left(V^{\vee}\right), \overline{\mathbb{Q}}_{\ell}\right)
$$

n'est autre que le foncteur

$$
K \mapsto \operatorname{pr}_{!}^{\vee}\left(\operatorname{pr}^{*} K \otimes J_{*} \overline{\mathbb{Q}}_{\ell}\right)[r-1]
$$

où $\mathrm{pr}^{\vee}$ et $\mathrm{pr}$ sont maintenant les deux projections canoniques de $\mathbb{P}\left(V^{\vee}\right) \times_{S} \mathbb{P}(V)$, et on a un dévissage fonctoriel en $K \in \mathrm{ob} D_{\mathrm{c}}^{\mathrm{b}}\left(\mathbb{P}(V), \overline{\mathbb{Q}}_{\ell}\right)$,

$$
\left(\bar{\pi}^{\vee 0}\right)^{*}\left(\bar{\pi}^{\circ}\right) ! K[r-1] \longrightarrow\left(j^{\vee}\right)^{*} \operatorname{Four}_{\mathcal{V} / S}(j ! K) \longrightarrow \operatorname{Rad}_{\mathbb{P}(V) / S}(K)(-1) \longrightarrow
$$

où $\bar{\pi}^{\vee \circ}: \mathbb{P}\left(V^{\vee}\right) \rightarrow S$ et $\bar{\pi}^{\circ}: \mathbb{P}(V) \rightarrow S$ sont les morphismes structuraux et où

$$
\operatorname{Rad}_{\mathbb{P}(V) / S}: D_{\mathrm{c}}^{\mathrm{b}}\left(\mathbb{P}(V), \overline{\mathbb{Q}}_{\ell}\right) \longrightarrow D_{\mathrm{c}}^{\mathrm{b}}\left(\mathbb{P}\left(V^{\vee}\right), \overline{\mathbb{Q}}_{\ell}\right)
$$

est la transformation de Radon géométrique (cf. [3]) définie par

$$
\operatorname{Rad}_{\mathbb{P}(V) / S}(K)=q ! q^{*} K[r-2]
$$

où $q^{\vee}: H \rightarrow \mathbb{P}\left(V^{\vee}\right)$ et $q: H \rightarrow \mathbb{P}(V)$ sont les restrictions à $H$ des deux projections canoniques.

Démonstration. - Comme la restriction du morphisme $\mu: \mathcal{V}^{\vee} \times{ }_{S} \mathcal{V} \rightarrow \mathcal{A}$ à l'ouvert $\mathbb{P}\left(V^{\vee}\right) \times{ }_{S} \mathbb{P}(V)$ est lisse, on a

$$
\left(j^{\vee} \times_{S} j\right)^{*} \mu^{*} \Psi \cong J_{*} \overline{\mathbb{Q}}_{\ell}
$$

par le théorème de changement de base par un morphisme lisse, d'où la première assertion.

Compte tenu de cette première assertion, on déduit du triangle distingué précédant l'énoncé de la proposition un triangle distingué

$$
\operatorname{pr!}_{!}^{\vee} \operatorname{pr}^{*} K[r-1] \rightarrow\left(j^{\vee}\right)^{*} \operatorname{Four}_{\mathcal{V} / S}(j ! K) \rightarrow \operatorname{pr}_{!}^{\vee}\left(\operatorname{pr}^{*} K \otimes I_{*} \overline{\mathbb{Q}}_{\ell}\right)[r-2](-1) \rightarrow,
$$

d'où la seconde assertion puisque $\operatorname{pr!̣}_{\text {! }}^{\vee} \mathrm{pr}^{*} \cong\left(\bar{\pi}^{\vee 0}\right)^{*}\left(\bar{\pi}^{\circ}\right)$ ! d'après le théorème de changement de base propre.

Soient $W \rightarrow S$ un deuxième fibré vectoriel de rang constant $s$ et $f: V \rightarrow W$ un morphisme de $S$-fibrés vectoriels de transposé ${ }^{\mathrm{t}} f: W^{\vee} \rightarrow V^{\vee}$. Par passage au quotient on a des $S$-morphismes, notés encore $f$ et ${ }^{\mathrm{t}} f$,

$$
f: \mathcal{V} \longrightarrow \mathcal{W} \text { et }{ }^{\mathrm{t}} f: \mathcal{W}^{\vee} \longrightarrow \mathcal{V}^{\vee}
$$

et on vérifie, comme on le fait pour la transformation de Fourier-Deligne [9], le lemme suivant.

LEMME 1.7. - On a un isomorphisme canonique de foncteurs

$$
\operatorname{Four}_{\mathcal{W} / S} \circ f_{!} \cong\left({ }^{\mathrm{t}} f\right)^{*} \circ \operatorname{Four}_{\mathcal{V} / S}[s-r]
$$


En particulier, si $i: B\left(\mathbb{G}_{\mathrm{m}, S}\right) \hookrightarrow \mathcal{V}$ et $i^{\vee}: B\left(\mathbb{G}_{\mathrm{m}, S}\right) \hookrightarrow \mathcal{V}^{\vee}$ sont les immersions fermées complémentaires des immersions ouvertes $j$ et $j^{\vee}$ et si on note encore $\pi: \mathcal{V} \rightarrow B\left(\mathbb{G}_{\mathrm{m}, S}\right)$ et $\pi^{\vee}: \mathcal{V}^{\vee} \rightarrow B\left(\mathbb{G}_{\mathrm{m}, S}\right)$ les morphismes représentables induits par les projections canoniques $\pi: V \rightarrow S$ et $\pi^{\vee}: V^{\vee} \rightarrow S$, on a

$$
\begin{aligned}
& \operatorname{Four}_{\mathcal{V} / S} \circ i_{!} \cong\left(\pi^{\vee}\right)^{*} \circ \operatorname{Four}_{B\left(\mathbb{G}_{\mathrm{m}, S}\right) / S}[r], \\
& \operatorname{Four}_{B\left(\mathbb{G}_{\mathrm{m}, S}\right) / S} \circ \pi_{!} \cong\left(i^{\vee}\right)^{*} \circ \operatorname{Four}_{\mathcal{V} / S}[-r] .
\end{aligned}
$$

Proposition 1.8. - La transformation de Fourier homogène

$$
\operatorname{Four}_{B\left(\mathbb{G}_{\mathrm{m}, S}\right) / S}: D_{\mathrm{c}}^{\mathrm{b}}\left(B\left(\mathbb{G}_{\mathrm{m}, S}\right), \overline{\mathbb{Q}}_{\ell}\right) \longrightarrow D_{\mathrm{c}}^{-}\left(B\left(\mathbb{G}_{\mathrm{m}, S}\right), \overline{\mathbb{Q}}_{\ell}\right)
$$

est le composé du foncteur $\sigma^{*}: D_{\mathrm{c}}^{\mathrm{b}}\left(B\left(\mathbb{G}_{\mathrm{m}, S}\right), \overline{\mathbb{Q}}_{\ell}\right) \rightarrow D_{\mathrm{c}}^{\mathrm{b}}\left(B\left(\mathbb{G}_{\mathrm{m}, S}\right), \overline{\mathbb{Q}}_{\ell}\right)$, où $\sigma: B\left(\mathbb{G}_{\mathrm{m}, S}\right) \rightarrow B\left(\mathbb{G}_{\mathrm{m}, S}\right)$ envoie $\mathcal{L}$ sur $\mathcal{L}^{\otimes-1}$, et du foncteur d'inclusion de $D_{\mathrm{c}}^{\mathrm{b}}\left(B\left(\mathbb{G}_{\mathrm{m}, S}\right), \overline{\mathbb{Q}}_{\ell}\right)$ dans $D_{\mathrm{c}}^{-}\left(B\left(\mathbb{G}_{\mathrm{m}, S}\right), \overline{\mathbb{Q}}_{\ell}\right)$.

Démonstration. - Par définition, on a

$$
\sigma^{*} \operatorname{Four}_{B\left(\mathbb{G}_{\mathrm{m}, S}\right) / S}(K)=\operatorname{pr}_{!}^{\vee}\left(\operatorname{pr}^{*} K \otimes \nu^{*} \alpha^{*} \Psi\right)[-1]
$$

où $\mathrm{pr}^{\vee}$ et pr sont les projections canoniques de $B\left(\mathbb{G}_{\mathrm{m}, S}\right) \times_{S} B\left(\mathbb{G}_{\mathrm{m}, S}\right)$ et où $\nu: B\left(\mathbb{G}_{\mathrm{m}, S}\right) \times_{S} B\left(\mathbb{G}_{\mathrm{m}, S}\right) \rightarrow B\left(\mathbb{G}_{\mathrm{m}, S}\right)$ envoie tout couple de fibrés en droites $(\mathcal{M}, \mathcal{L})$ sur le fibré en droites $\mathcal{L} \otimes \mathcal{M}^{\otimes-1}$. De plus, on a un isomorphisme

$$
\alpha^{*} \Psi \stackrel{\sim}{\longrightarrow} g_{!} \overline{\mathbb{Q}}_{\ell}[1]
$$

d'après 1.4 (ii). Enfin, on a le carré cartésien

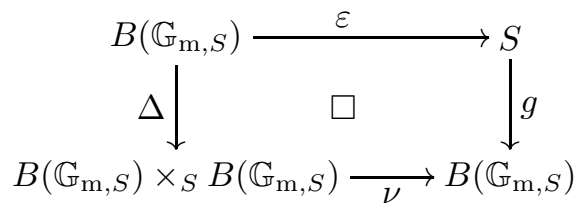

où $\Delta$ est le morphisme morphisme diagonal et $\varepsilon$ est le morphisme structural.

Appliquant le théorème de changement de base propre à ce carré et la formule des projections, on obtient que

$$
\operatorname{pr}_{!}^{\vee}\left(\operatorname{pr}^{*} K \otimes \nu^{*} g_{!} \overline{\mathbb{Q}}_{\ell}\right)=\operatorname{pr}_{!}^{\vee}\left(\operatorname{pr}^{*} K \otimes \Delta_{!} \overline{\mathbb{Q}}_{\ell}\right)=K,
$$

d'où la proposition.

Corollaire 1.9. - L'image essentielle de la transformation de Fourier homogène $\operatorname{Four}_{\mathcal{V} / S}$ est contenue dans la sous-catégorie pleine $D_{\mathrm{c}}^{\mathrm{b}}\left(\mathcal{V}^{\vee} / S, \overline{\mathbb{Q}}_{\ell}\right)$ de $D_{\mathrm{c}}^{-}\left(\mathcal{V}^{\vee} / S, \overline{\mathbb{Q}}_{\ell}\right)$.

Démonstration. - Tout $K \in \mathrm{ob} D_{\mathrm{c}}^{\mathrm{b}}\left(\mathcal{V} / S, \overline{\mathbb{Q}}_{\ell}\right)$ admet le dévissage

$$
j_{!} j^{*} K \longrightarrow K \longrightarrow i_{*} i^{*} K \longrightarrow,
$$

de sorte que $\operatorname{Four}_{\mathcal{V} / S}(K)$ admet le dévissage

$$
\operatorname{Four}_{\mathcal{V} / S}\left(j ! j^{*} K\right) \longrightarrow \operatorname{Four}_{\mathcal{V} / S}(K) \longrightarrow \operatorname{Four}_{\mathcal{V} / S}\left(i_{!} i^{*} K\right) \longrightarrow
$$


et que $\operatorname{Four}_{\mathcal{V} / S}\left(j ! j^{*} K\right)$ admet à son tour le dévissage

$$
j_{!}^{\vee}\left(j^{\vee}\right)^{*} \operatorname{Four}_{\mathcal{V} / S}\left(j ! j^{*} K\right) \rightarrow \operatorname{Four}_{\mathcal{V} / S}\left(j ! j^{*} K\right) \rightarrow i_{*}^{\vee}\left(i^{\vee}\right)^{*} \operatorname{Four}_{\mathcal{V} / S}\left(j ! j^{*} K\right) \rightarrow .
$$

D'après la proposition 1.6, $\left(j^{\vee}\right)^{*} \operatorname{Four}_{\mathcal{V} / S}\left(j ! j^{*} K\right)$ est à cohomologie bornée et, d'après le lemme 1.7 et la proposition 1.8 , il en est de même de

$$
\operatorname{Four}_{\mathcal{V} / S}\left(i_{!} i^{*} K\right)=\left(\pi^{\vee}\right)^{*} \operatorname{Four}_{B\left(\mathbb{G}_{\mathrm{m}, S}\right) / S}\left(i^{*} K\right)[r]=\left(\pi^{\vee}\right)^{*} \sigma^{*} i^{*} K[r]
$$

et de

$$
\left(i^{\vee}\right)^{*} \operatorname{Four}_{\mathcal{V} / S}\left(j ! j^{*} K\right)=\operatorname{Four}_{B\left(\mathbb{G}_{\mathrm{m}, S}\right) / S}\left(\pi_{!} j_{!} j^{*} K\right)=\sigma^{*} \pi_{!}^{\circ} j^{*} K[r]
$$

où $\pi^{\circ}=\pi \circ j: \mathbb{P}(V) \rightarrow B\left(\mathbb{G}_{\mathrm{m}, S}\right)$, d'où le corollaire.

\section{Relation entre les transformations de Fourier homogène et de Fourier-Deligne}

2.1. Supposons dans cette section que $S$ est de caractéristique $p>0$ et fixons un caractère additif non trivial $\psi: \mathbb{F}_{p} \rightarrow \overline{\mathbb{Q}}_{\ell}^{\times}$.

La transformation de Fourier-Deligne associée à $V / S$ et à $\psi$ (cf. [8] et [9]) est le foncteur

$$
\operatorname{Four}_{V / S, \psi}: D_{\mathrm{c}}^{\mathrm{b}}\left(V, \overline{\mathbb{Q}}_{\ell}\right) \longrightarrow D_{\mathrm{c}}^{\mathrm{b}}\left(V^{\vee}, \overline{\mathbb{Q}}_{\ell}\right)
$$

défini par

$$
\operatorname{Four}_{V / S, \psi}(K)=\operatorname{pr}_{!}^{\vee}\left(\operatorname{pr}^{*} K \otimes\langle,\rangle^{*} \mathcal{L}_{\psi}\right)[r]
$$

où $\operatorname{pr}^{\vee}$ et pr sont les projections canoniques de $V^{\vee} \times{ }_{S} V$, où

$$
\langle,\rangle: V^{\vee} \times_{S} V \longrightarrow \mathbb{A}_{S}^{1}
$$

est l'accouplement canonique et où $\mathcal{L}_{\psi}$ est le $\overline{\mathbb{Q}}_{\ell}$-faisceau lisse de rang 1 d'ArtinSchreier sur $\mathbb{A}_{S}^{1}$ associé au caractère $\psi$.

ThÉORÈME 2.2. - Les foncteurs composés

$$
\left(\rho^{\vee}\right)^{*} \circ \operatorname{Four}_{\mathcal{V} / S} \text { et } \operatorname{Four}_{V / S, \psi} \circ \rho^{*}: D_{\mathrm{c}}^{\mathrm{b}}\left(\mathcal{V}, \overline{\mathbb{Q}}_{\ell}\right) \longrightarrow D_{\mathrm{c}}^{\mathrm{b}}\left(V^{\vee}, \overline{\mathbb{Q}}_{\ell}\right)
$$

sont canoniquement isomorphes.

Démonstration. - Faisons agir $\mathbb{G}_{\mathrm{m}, S}$ sur $V^{\vee} \times{ }_{S} V$ de manière anti-diagonale $\left(t \cdot(w, v)=\left(t^{-1} w, t v\right)\right)$. On a le diagramme à carrés cartésiens

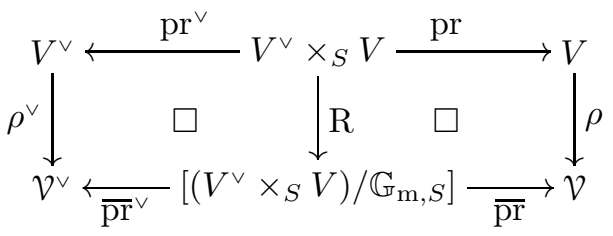


où R est le morphisme quotient, et $\langle\rangle:, V^{\vee} \times_{S} V \rightarrow \mathbb{A}_{S}^{1}$ se factorise en

$$
V^{\vee} \times{ }_{S} V \stackrel{\mathrm{R}}{\longrightarrow}\left[\left(V^{\vee} \times{ }_{S} V\right) / \mathbb{G}_{\mathrm{m}, S}\right] \stackrel{\overline{\langle,\rangle}}{\longrightarrow} \mathbb{A}_{S}^{1}
$$

Une application du théorème de changement de base pour un morphisme représentable propre montre que, pour tout $K \in$ ob $D_{\mathrm{c}}^{\mathrm{b}}\left(\mathcal{V}, \overline{\mathbb{Q}}_{\ell}\right)$

$$
\operatorname{Four}_{V / S, \psi}\left(\rho^{*} K\right)=\left(\rho^{\vee}\right)^{*} \overline{\operatorname{pr}}_{!}^{\vee}\left(\overline{\operatorname{pr}}^{*} K \otimes \overline{\langle,\rangle^{*}} \mathcal{L}_{\psi}\right)[r] .
$$

En termes imagés, le transformé de Fourier-Deligne d'un complexe $\mathbb{G}_{\mathrm{m}, S^{-}}$ équivariant est aussi $\mathbb{G}_{\mathrm{m}, S}$-équivariant.

Maintenant, $\mathcal{V}^{\vee} \times{ }_{S} \mathcal{V}$ est le quotient de $\left[\left(V^{\vee} \times{ }_{S} V\right) / \mathbb{G}_{\mathrm{m}, S}\right]$ par l'action de $\mathbb{G}_{\mathrm{m}, S}$ induite par celle par homothétie sur le facteur $V$ de $V^{\vee} \times{ }_{S} V$. Si on note

$$
F:\left[\left(V^{\vee} \times{ }_{S} V\right) / \mathbb{G}_{\mathrm{m}, S}\right] \longrightarrow \mathcal{V}^{\vee} \times{ }_{S} \mathcal{V}
$$

le morphisme quotient, les morphismes $\overline{\mathrm{pr}}$ et $\overline{\mathrm{pr}}^{\vee}$ introduits ci-dessus se factorisent par $F$ en les projections canoniques pr et $\mathrm{pr}^{\vee}$ de $\mathcal{V}^{\vee} \times_{S} \mathcal{V}$. Par suite, une application de la formule des projections nous donne un isomorphisme canonique

$$
\overline{\operatorname{pr}}_{!}^{\vee}\left(\overline{\operatorname{pr}}^{*} K \otimes \overline{\langle,\rangle}^{*} \mathcal{L}_{\psi}\right)[r] \cong \operatorname{pr}_{!}^{\vee}\left(\operatorname{pr}^{*} K \otimes F_{!} \overline{\langle,\rangle^{*}} \mathcal{L}_{\psi}\right)[r]
$$

Calculons enfin $F_{!} \overline{\langle,\rangle} \mathcal{L}_{\psi}$. On a le carré cartésien

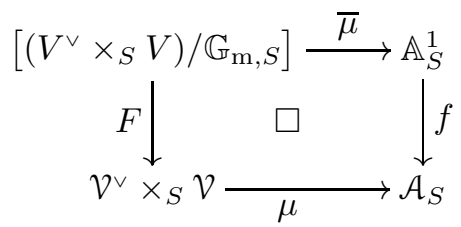

où $f: \mathbb{A}_{S}^{1} \rightarrow\left[\mathbb{A}_{S}^{1} / \mathbb{G}_{\mathrm{m}, S}\right]=\mathcal{A}_{S}$ est le morphisme quotient. En appliquant de nouveau le théorème de changement de base propre, on obtient donc un isomorphisme canonique

$$
F ! \overline{\langle,\rangle}^{*} \mathcal{L}_{\psi} \cong \mu^{*} f_{!} \mathcal{L}_{\psi}
$$

On conclut par le lemme 2.3 ci-dessous.

LEMME 2.3. - Le complexe $\beta^{*} f_{!} \mathcal{L}_{\psi}$ est canoniquement isomorphe au faisceau constant $\overline{\mathbb{Q}}_{\ell}$ sur $S$, placé en degré 1 , et le morphisme d'adjonction $f_{!} \mathcal{L}_{\psi} \rightarrow \beta_{*} \beta^{*} f_{!} \mathcal{L}_{\psi}$ est un isomorphisme. Par suite, le complexe $f_{!} \mathcal{L}_{\psi}[1]$ est canoniquement isomorphe au complexe $\Psi=\beta_{*} \overline{\mathbb{Q}}_{\ell}$ défini en 1.3 .

Démonstration. — Si $h: \mathbb{A}_{S}^{1} \rightarrow S$ est le morphisme structural, il est bien connu que $h_{!} \mathcal{L}_{\psi}=(0)$. Par suite, $\beta^{*} f_{!} \mathcal{L}_{\psi}$, qui est l'image directe par le morphisme structural $\mathbb{G}_{\mathrm{m}, S} \rightarrow S$ de la restriction à $\mathbb{G}_{\mathrm{m}, S} \subset \mathbb{A}_{S}^{1}$ de $\mathcal{L}_{\psi}$, est canoniquement isomorphe à $\overline{\mathbb{Q}}_{\ell}[-1]$.

BULLETIN DE LA SOCIÉTÉ MATHÉMATIQUE DE FRANCE 
Le cône de la flèche d'adjonction $f_{!} \mathcal{L}_{\psi} \rightarrow \beta_{*} \beta^{*} f_{!} \mathcal{L}_{\psi}$ est le complexe $\alpha^{!} f_{!} \mathcal{L}_{\psi}[1]$ et il nous reste à démontrer que $\alpha^{!} f_{!} \mathcal{L}_{\psi}=(0)$, ou ce qui revient au même par dualité, que $\alpha^{*} f_{*} \mathcal{L}_{\psi^{-1}}=(0)$. Pour cela, on peut se restreindre à $\mathbb{A}_{S}^{1}$ par le morphisme lisse $f: \mathbb{A}_{S}^{1} \rightarrow \mathcal{A}_{S}$. Comme le carré

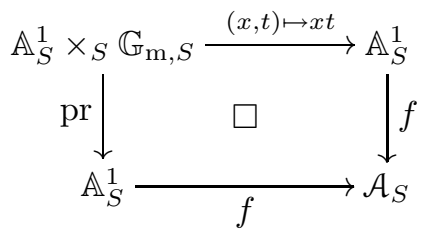

est cartésien, une application du théorème de changement de base lisse nous ramène donc à vérifier que $0^{*} \mathrm{pr}_{*} \mathcal{L}_{\psi^{-1}}(x t)=(0)$, où pr est la projection canonique sur le premier facteur de $\mathbb{A}_{S}^{1} \times{ }_{S} \mathbb{G}_{\mathrm{m}, S}$ et $0: S \rightarrow \mathbb{A}_{S}^{1}$ est la section nulle.

Compactifions pr en

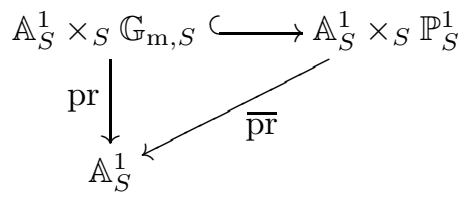

le fermé complémentaire de $\mathbb{A}_{S}^{1} \times_{S} \mathbb{G}_{\mathrm{m}, S}$ dans $\mathbb{A}_{S}^{1} \times_{S} \mathbb{P}_{S}^{1}$ étant la réunion disjointe des images des deux sections évidentes $\mathbb{A}_{S}^{1} \hookrightarrow \mathbb{A}_{S}^{1} \times_{S} \mathbb{P}_{S}^{1}, x \mapsto(x, 0)$ et $x \mapsto(x, \infty)$.

Rappelons que le système local $\mathcal{L}_{\psi^{-1}}(x t)$ sur $\mathbb{A}_{S}^{1} \times_{S} \mathbb{G}_{\mathrm{m}, S} \subset \mathbb{A}_{S}^{1} \times_{S} \mathbb{P}_{S}^{1}$ se prolonge en un système local, encore noté $\mathcal{L}_{\psi^{-1}}(x t)$, sur $\mathbb{A}_{S}^{1} \times_{S} \mathbb{A}_{S}^{1} \subset \mathbb{A}_{S}^{1} \times_{S} \mathbb{P}_{S}^{1}$, et que les prolongements par zéro et par image directe totale de ce dernier système local à $\mathbb{A}_{S}^{1} \times{ }_{S} \mathbb{P}_{S}^{1}$ tout entier coïncident (cf. la démonstration de (2.4.1) dans [8]).

Si on note $\overline{\mathcal{L}}_{\psi^{-1}}(x t)$ ces deux mêmes prolongements, on a un triangle distingué

$$
\overline{\mathrm{pr}}_{*} \overline{\mathcal{L}}_{\psi^{-1}}(x t) \longrightarrow \operatorname{pr}_{*} \mathcal{L}_{\psi^{-1}}(x t) \longrightarrow \overline{\mathbb{Q}}_{\ell}[-1](-1) \longrightarrow
$$

dans $D_{\mathrm{c}}^{\mathrm{b}}\left(\mathbb{A}_{S}^{1}, \overline{\mathbb{Q}}_{\ell}\right)$, et donc un triangle distingué

$$
0^{*} \overline{\mathrm{pr}}_{*} \overline{\mathcal{L}}_{\psi^{-1}}(x t) \longrightarrow 0^{*} \operatorname{pr}_{*} \mathcal{L}_{\psi^{-1}}(x t) \longrightarrow \overline{\mathbb{Q}}_{\ell}[-1](-1) \longrightarrow
$$

dans $D_{\mathrm{c}}^{\mathrm{b}}\left(S, \overline{\mathbb{Q}}_{\ell}\right)$, et il ne reste plus qu'à vérifier que le flèche de bord

$$
\overline{\mathbb{Q}}_{\ell}[-1](-1) \longrightarrow 0^{*} \overline{\mathrm{pr}}_{*} \overline{\mathcal{L}}_{\psi^{-1}}(x t)[1]
$$

est un isomorphisme. Mais, par le théorème de changement de base propre, cette flèche n'est autre, à un décalage près, que l'inverse de l'isomorphisme trace

$$
h_{!} \overline{\mathbb{Q}}_{\ell} \stackrel{\sim}{\longrightarrow} \overline{\mathbb{Q}}_{\ell}[-2](-1),
$$

TOME $131-2003-\mathrm{N}^{\mathrm{O}} 4$ 
d'où la conclusion.

Remarque 2.3.1. - Comme S. Lysenko me l'a fait remarquer, on peut démontrer le lemme 2.3 en utilisant le lemme d'homotopie 5.5 plus loin dans cet article.

En effet, on peut l'appliquer au fibré vectoriel $h: \mathcal{A}_{S} \rightarrow B\left(\mathbb{G}_{\mathrm{m}, S}\right)$, à sa section nulle $\alpha: B\left(\mathbb{G}_{\mathrm{m}, S}\right) \hookrightarrow \mathcal{A}_{S}$ et au complexe $\ell$-adique $\mathbb{G}_{\mathrm{m}, S \text {-équivariant }}$ $f_{*} \mathcal{L}_{\psi^{-1}}$ sur $\mathcal{A}_{S}$. On a donc

$$
\alpha^{*} f_{*} \mathcal{L}_{\psi^{-1}} \cong h_{*} f_{*} \mathcal{L}_{\psi^{-1}}=g_{*} h_{*} \mathcal{L}_{\psi^{-1}}=(0)
$$

où les flèches $h$ et $g$ dans l'expression $g_{*} h_{*} \mathcal{L}_{\psi^{-1}}$ sont $h: \mathbb{A}_{S}^{1} \rightarrow S$ et $g: S \rightarrow B\left(\mathbb{G}_{\mathrm{m}, S}\right)$.

2.4. Il résulte du théorème 2.2 que, si $S$ est de caractéristique $p>0$, la transformation de Fourier homogène est involutive, commute à la dualité et induit une équivalence de la catégorie des $\overline{\mathbb{Q}}_{\ell}$-faisceaux pervers sur $V$ sur celle des $\overline{\mathbb{Q}}_{\ell}$-faisceaux pervers sur $\mathcal{V}^{\vee}$. Dans les deux sections qui suivent, nous allons donner une démonstration directe de ces propriétés et donc voir qu'elles valent aussi pour $S$ arbitraire.

\section{Involutivité de la transformation de Fourier homogène}

ThÉORÈme 3.1. - La transformation de Fourier homogène est involutive au sens où le foncteur composé

$$
\operatorname{Four}_{\mathcal{V} \vee / S} \circ \operatorname{Four}_{\mathcal{V} / S}: D_{\mathrm{c}}^{\mathrm{b}}\left(\mathcal{V}, \overline{\mathbb{Q}}_{\ell}\right) \longrightarrow D_{\mathrm{c}}^{\mathrm{b}}\left(\mathcal{V}, \overline{\mathbb{Q}}_{\ell}\right)
$$

est canoniquement isomorphe au foncteur $K \mapsto K(-r)$.

Avant de démontrer le théorème, établissons quelques propriétés du complexe $\Psi=\beta_{*} \overline{\mathbb{Q}}_{\ell} \in D_{\mathrm{c}}^{\mathrm{b}}\left(\mathcal{A}_{S}, \overline{\mathbb{Q}}_{\ell}\right)$.

Lemme 3.2. - Soient $f: \mathbb{A}_{S}^{1} \rightarrow \mathcal{A}_{S}$ le morphisme quotient et $v: \mathbb{A}_{S}^{1}-a(S) \hookrightarrow$ $\mathbb{A}_{S}^{1}$ l'ouvert complémentaire de l'image d'une section $a: S \rightarrow \mathbb{A}_{S}^{1}$ partout non nulle. Le complexe $\beta^{*} f_{!} v_{*} \overline{\mathbb{Q}}_{\ell}$ est canoniquement isomorphe au faisceau constant $\overline{\mathbb{Q}}_{\ell}$ sur $S$, placé en degré 1 , et le morphisme d'adjonction $f_{!} v_{*} \overline{\mathbb{Q}}_{\ell} \rightarrow \beta_{*} \beta^{*} f_{!} v_{*} \overline{\mathbb{Q}}_{\ell}$ est un isomorphisme. Par suite, le complexe $f_{!} v_{*} \overline{\mathbb{Q}}_{\ell}[1]$ est canoniquement isomorphe au complexe $\Psi=\beta_{*} \overline{\mathbb{Q}}_{\ell}$ défini en 1.3 .

Démonstration. - Le complexe $\beta^{*} f_{!} v_{*} \overline{\mathbb{Q}}_{\ell}$ est l'image directe à supports propres par le morphisme structural $\mathbb{G}_{\mathrm{m}, S} \rightarrow S$ du faisceau constant $\overline{\mathbb{Q}}_{\ell}$ sur $\mathbb{G}_{\mathrm{m}, S}-a(S)$ prolongé par image directe totale à $\mathbb{G}_{\mathrm{m}, S}$ tout entier. On a donc bien $\beta^{*} f_{!} v_{*} \overline{\mathbb{Q}}_{\ell} \cong \overline{\mathbb{Q}}_{\ell}[-1]$ d'après l'assertion (i) du lemme 1.4.

En raisonnant comme dans la démonstration du lemme 2.3, on voit qu'il nous reste à vérifier que $0^{*} \mathrm{pr}_{*} w ! \overline{\mathbb{Q}}_{\ell}=(0)$ où $w$ est l'inclusion dans $\mathbb{A}_{S}^{1} \times_{S} \mathbb{G}_{\mathrm{m}, S}$ de l'ouvert complémentaire du fermé d'équation $x t=a$.

BULLETIN DE LA SOCIÉTÉ MATHÉMATIQUE DE FRANCE 
Considérons la compactification partielle

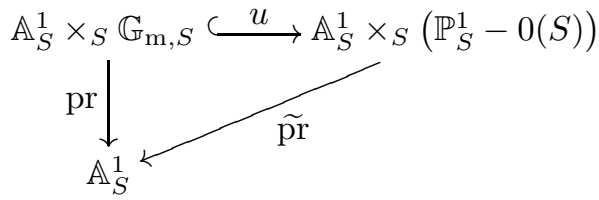

le fermé complémentaire de $\mathbb{A}_{S}^{1} \times{ }_{S} \mathbb{G}_{\mathrm{m}, S}$ dans $\mathbb{A}_{S}^{1} \times_{S}\left(\mathbb{P}_{S}^{1}-0(S)\right)$ étant $\mathbb{A}_{S}^{1} \times_{S}$ $\infty(S)$. Si $y=t^{-1}$ est la coordonnée affine à l'infini de $\mathbb{P}_{S}^{1}$, le fermé d'équation $x t=a$ de $\mathbb{A}_{S}^{1} \times_{S} \mathbb{G}_{\mathrm{m}, S}$ se prolonge en le fermé d'équation $x=a y$ de $\mathbb{A}_{S}^{1} \times_{S}$ $\left(\mathbb{P}_{S}^{1}-0(S)\right)=S[x, y]$, fermé qui est transverse au fermé $\mathbb{A}_{S}^{1} \times_{S} \infty(S)$ d'équation $y=0$. Si on note $\widetilde{w}$ l'inclusion de l'ouvert $\{x \neq a y\}$ dans $\mathbb{A}_{S}^{1} \times_{S}\left(\mathbb{P}_{S}^{1}-0(S)\right)$ et $\widetilde{u}$ l'inclusion de

$$
\left\{(x, y) \in \mathbb{A}_{S}^{1} \times_{S} \mathbb{G}_{\mathrm{m}, S} \mid x \neq a y\right\}
$$

dans

$$
\left\{(x, y) \in \mathbb{A}_{S}^{1} \times_{S}\left(\mathbb{P}_{S}^{1}-0(S)\right) \mid x \neq a y\right\},
$$

on a

$$
u_{*} w_{!}=\widetilde{w}_{!} \widetilde{u}_{*} \overline{\mathbb{Q}}_{\ell}
$$

par la formule de Künneth, et on a donc un triangle distingué

$$
\widetilde{\mathrm{pr}}_{*} \widetilde{w}_{!} \overline{\mathbb{Q}}_{\ell} \longrightarrow \operatorname{pr}_{*} w_{!} \overline{\mathbb{Q}}_{\ell} \longrightarrow \widetilde{\mathrm{pr}}_{*} \widetilde{w}_{!} R^{1} \widetilde{u}_{*} \overline{\mathbb{Q}}_{\ell}[-1] \longrightarrow .
$$

Or, on a

$$
\widetilde{\mathrm{pr}}_{*} \widetilde{w}_{!} \overline{\mathbb{Q}}_{\ell}=(0)
$$

d'après 1.4(i), et $R^{1} \widetilde{u}_{*} \overline{\mathbb{Q}}_{\ell}$ est la faisceau $\overline{\mathbb{Q}}_{\ell}(-1)$ sur la partie localement fermée $\mathbb{G}_{\mathrm{m}, S} \times_{S} \infty(S)=\left\{(x, y) \in \mathbb{A}_{S}^{1} \times_{S}\left(\mathbb{P}_{S}^{1}-0(S)\right) \mid x \neq 0, y=0\right\}$, de sorte que $\widetilde{\mathrm{pr}}_{*} \widetilde{w}_{!} R^{1} \widetilde{u}_{*} \overline{\mathbb{Q}}_{\ell}[-1]$ est le prolongement par zéro de $\overline{\mathbb{Q}}_{\ell}[-1](-1)$ de $\mathbb{G}_{\mathrm{m}, S}$ à $\mathbb{A}_{S}^{1}$. Par suite, on a bien $0^{*} \operatorname{pr}_{*} w_{!} \overline{\mathbb{Q}}_{\ell}=(0)$ et le lemme est démontré.

Remarque 3.2.1. - Comme me l'a signalé S. Lysenko, le lemme 3.2 résulte aussi du lemme d'homotopie 5.5 et de l'assertion (i) de 1.4 (cf. remarque 2.3.1).

Lemme 3.3. - On a

$$
\bar{h}_{!} \Psi=(0)
$$

où $\bar{h}: \mathcal{A}_{S} \rightarrow S$ est le morphisme structural.

Démonstration. - Cela résulte immédiatement des lemmes 3.2 et 1.4 (i) puisque $h=\bar{h} \circ f$.

Lemme 3.4. - Faisons agir $\mathbb{G}_{\mathrm{m}, S}$ diagonalement par homothétie sur $\mathbb{A}_{S}^{2}$ et soient $q:\left[\mathbb{A}_{S}^{2} / \mathbb{G}_{\mathrm{m}, S}\right] \rightarrow \mathcal{A}_{S}^{2}=\mathcal{A}_{S} \times_{S} \mathcal{A}_{S}$ le morphisme quotient et $\sigma:$ $\left[\mathbb{A}_{S}^{2} / \mathbb{G}_{\mathrm{m}, S}\right] \rightarrow \mathcal{A}_{S}$ le morphisme induit par le morphisme différence $\mathbb{A}_{S}^{2} \rightarrow \mathbb{A}_{S}^{1}$, $(x, y) \mapsto x-y$. Alors on a un isomorphisme canonique

$$
q ! \sigma^{*} \Psi[1] \cong \Psi \otimes \Psi .
$$


Démonstration. — On a d'une part

$$
\sigma^{*} \beta_{*} \overline{\mathbb{Q}}_{\ell}=j_{*} \overline{\mathbb{Q}}_{\ell}
$$

où $j: \mathcal{U} \hookrightarrow\left[\mathbb{A}_{S}^{2} / \mathbb{G}_{\mathrm{m}, S}\right]$ est l'ouvert quotient de l'ouvert $\{(x, y) \mid x \neq y\}$ de $\mathbb{A}_{S}^{2}$, puisque $\sigma$ est représentable et lisse. On a d'autre part

$$
\beta_{*} \overline{\mathbb{Q}}_{\ell} \otimes \beta_{*} \overline{\mathbb{Q}}_{\ell} \cong\left(\beta^{2}\right)_{*} \overline{\mathbb{Q}}_{\ell}
$$

où $\beta^{2}$ est l'immersion ouverte $\beta \times \beta: S=S \times{ }_{S} S \hookrightarrow \mathcal{A}_{S}^{2}$, d'après la formule de Künneth. On cherche donc à construire un isomorphisme canonique

$$
q_{!} j_{*} \overline{\mathbb{Q}}_{\ell}[1] \stackrel{\sim}{\longrightarrow}\left(\beta^{2}\right)_{*} \overline{\mathbb{Q}}_{\ell}
$$

L'immersion ouverte $\beta^{2}$ se factorise en

$$
S \stackrel{\gamma}{\longrightarrow} \mathcal{B} \stackrel{i}{\longrightarrow} \mathcal{A}_{S}^{2}
$$

où $i: \mathcal{B} \hookrightarrow \mathcal{A}_{S}^{2}$ est l'image de l'ouvert $\mathbb{A}_{S}^{2}-\{(0,0)\} \subset \mathbb{A}_{S}^{2}$. Il suffit donc :

1) de construire un isomorphisme canonique $i^{*} q_{!} j_{*} \overline{\mathbb{Q}}_{\ell}[1] \stackrel{\sim}{\longrightarrow} \gamma_{*} \overline{\mathbb{Q}}_{\ell}$,

2) de montrer que la flèche d'adjonction $q_{!} j_{*} \overline{\mathbb{Q}}_{\ell} \rightarrow i_{*} i^{*} q_{!} j_{*} \overline{\mathbb{Q}}_{\ell}$ est un isomorphisme.

Le champ $\mathcal{B}$ admet le recouvrement ouvert $\mathcal{B}=\mathcal{B}_{1} \cup \mathcal{B}_{2}$ où $\mathcal{B}_{1}=\mathcal{A}_{S} \times_{S} S$ et $\mathcal{B}_{2}=S \times{ }_{S} \mathcal{A}_{S}$ sont tous les deux isomorphes à $\mathcal{A}_{S}$, et la restriction du morphisme composé

$$
\mathcal{U} \stackrel{j}{\longrightarrow}\left[\mathbb{A}_{S}^{2} / \mathbb{G}_{\mathrm{m}, S}\right] \stackrel{q}{\longrightarrow} \mathcal{A}_{S}^{2}
$$

à l'un ou l'autre de ces ouverts est naturellement isomorphe à

$$
\mathbb{A}_{S}^{1}-\{1(S)\} \stackrel{v}{\longrightarrow} \mathbb{A}_{S}^{1} \stackrel{f}{\longrightarrow} \mathcal{A}_{S}
$$

où $1: S \rightarrow \mathbb{A}_{S}^{1}$ est la section constante de valeur 1 . Par suite, la restriction de $i^{*} q_{!} j_{*} \overline{\mathbb{Q}}_{\ell}[1]$ à $\mathcal{B}_{1} \cong \mathcal{A}_{S}$ ou $\mathcal{B}_{2} \cong \mathcal{A}_{S}$ est canoniquement isomorphe à $f_{*} v_{!} \overline{\mathbb{Q}}_{\ell}[1]$, et donc à $\beta_{*} \overline{\mathbb{Q}}_{\ell}$, d'après le lemme 3.2 , d'où le point 1 ).

Il suffit de démontrer le point 2) après le changement de base par le morphisme quotient $\mathbb{A}_{S}^{2} \rightarrow \mathcal{A}_{S}^{2}$. Par suite, d'après le théorème de changement de base par un morphisme lisse, si on note encore $j$ l'inclusion de l'ouvert

$$
U=\{(x, y, t) \mid x t \neq y\}
$$

dans $\mathbb{A}_{S}^{2} \times{ }_{S} \mathbb{G}_{\mathrm{m}, S}, q$ la projection canonique de $\mathbb{A}_{S}^{2} \times{ }_{S} \mathbb{G}_{\mathrm{m}, S}$ sur $\mathbb{A}_{S}^{2}$ et $i$ l'inclusion de $\mathbb{A}_{S}^{2}-\{(0,0)\}$ dans $\mathbb{A}_{S}^{2}$, il ne nous reste plus qu'à démontrer que la flèche d'adjonction $q_{!} j_{*} \overline{\mathbb{Q}}_{\ell} \rightarrow i_{*} i^{*} q_{!} j_{*} \overline{\mathbb{Q}}_{\ell}$ est un isomorphisme dans $D_{\mathrm{c}}^{\mathrm{b}}\left(\mathbb{A}_{S}^{2}, \overline{\mathbb{Q}}_{\ell}\right)$. 
On compactifie la projection $q$ en

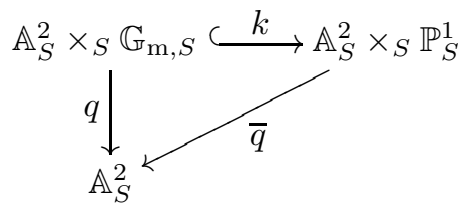

et on introduit l'ouvert

$$
\bar{\jmath}: \bar{U}=\{((x, y),(T ; U)) \mid x T-y V \neq 0\} \longleftrightarrow \mathbb{A}_{S}^{2} \times{ }_{S} \mathbb{P}_{S}^{1} .
$$

On a un carré cartésien d'immersions ouvertes

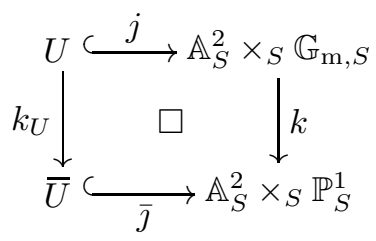

et, par adjonction, une flèche

$$
k_{!} j_{*} \overline{\mathbb{Q}}_{\ell} \longrightarrow \bar{j}_{*} k_{U, !} \overline{\mathbb{Q}}_{\ell} .
$$

Cette flèche est un isomorphisme. En effet, le problème est local sur $\mathbb{A}_{S}^{2} \times{ }_{S} \mathbb{P}_{S}^{1}$ en les points du fermé complémentaire de la réunion des deux ouverts $\mathbb{A}_{S}^{2} \times{ }_{S} \mathbb{A}_{S}^{1}$ et $\bar{U}$. Or, en ces points on est localement dans une situation produit. En effet, on a des coordonnées $(x, y,(T ; U))$ sur $\mathbb{A}_{S}^{2} \times{ }_{S} \mathbb{P}_{S}^{1}, \mathbb{A}_{S}^{2} \times{ }_{S} \mathbb{A}_{S}^{1}$ est le complémentaire du diviseur lisse $\{(T ; U)=(0 ; 1)\} \cup\{(T ; U)=(1 ; 0)\}, \bar{U}$ est le complémentaire du diviseur lisse $\{x T-y U=0\}$ et ces deux diviseurs lisses se coupent transversalement. On conclut donc par la formule de Künneth.

On a donc des isomorphismes canoniques

$$
q_{!} j_{*} \overline{\mathbb{Q}}_{\ell} \cong \bar{q}_{*} k_{!} j_{*} \overline{\mathbb{Q}}_{\ell} \cong \bar{q}_{*} \bar{j}_{*} k_{U, !} \overline{\mathbb{Q}}_{\ell}
$$

et, comme $\bar{q} \circ \bar{\jmath}$ se factorise par $i$ en

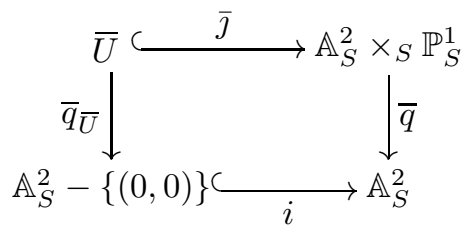

on a en fait un isomorphisme canonique

$$
q_{!} j_{*} \overline{\mathbb{Q}}_{\ell} \cong i_{*} \bar{q}_{\bar{U}, *} k_{U, !} \overline{\mathbb{Q}}_{\ell},
$$

d'où le point 2). 
Remarque 3.4.1. - Toujours comme me l'a fait remarquer S. Lysenko, l'assertion 2) de la preuve ci-dessus peut aussi se déduire du lemme d'homotopie 5.5 appliqué au fibré vectoriel $\mathcal{A}_{S} \times{ }_{S} \mathcal{A}_{S} \rightarrow B\left(\mathbb{G}_{\mathrm{m}, S}\right) \times{ }_{S} B\left(\mathbb{G}_{\mathrm{m}, S}\right)$ et au complexe $\ell$-adique $\mathbb{G}_{\mathrm{m}, S}$-équivariant $q_{!} j_{*} \overline{\mathbb{Q}}_{\ell}$.

Démonstration du théorème 3.1. - On procède comme pour la transformation de Fourier-Deligne (cf. [9], (1.2.2)). Il s'agit donc de construire un isomorphisme

$$
\operatorname{pr}_{13, !} \nu^{*}(\Psi \otimes \Psi) \cong \Delta_{!} \overline{\mathbb{Q}}_{\ell}[2-2 r](-r)
$$

où $\operatorname{pr}_{13}: \mathcal{V} \times_{S} \mathcal{V}^{\vee} \times_{S} \mathcal{V} \rightarrow \mathcal{V} \times_{S} \mathcal{V}$ est la projection canonique, où

$$
\nu: \mathcal{V} \times{ }_{S} \mathcal{V}^{\vee} \times{ }_{S} \mathcal{V} \longrightarrow \mathcal{A}_{S}^{2}
$$

est induit par le morphisme $\left(v_{1}, w, v_{2}\right) \mapsto\left(\left\langle w, v_{1}\right\rangle,\left\langle w, v_{2}\right\rangle\right)$ de $V \times_{S} V^{\vee} \times_{S} V$ dans $\mathbb{A}_{S}^{2}$ et où $\Delta: \mathcal{V} \rightarrow \mathcal{V} \times_{S} \mathcal{V}$ est le morphisme diagonal.

On a le carré cartésien

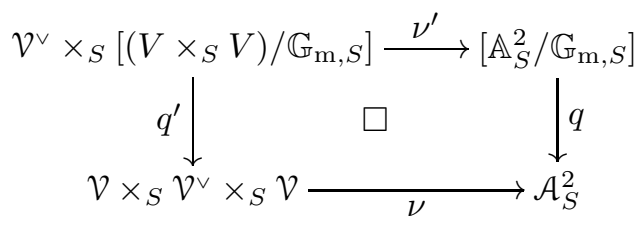

où $\nu^{\prime}$ est induite par le morphisme de $V^{\vee} \times_{S} V \times_{S} V$ dans $\mathbb{A}_{S}^{2}$ qui envoie $\left(w, v_{1}, v_{2}\right)$ sur $\left(\left\langle w, v_{1}\right\rangle,\left\langle w, v_{2}\right\rangle\right)$ et $q^{\prime}$ est induite par le morphisme de $V^{\vee} \times_{S}$ $V \times{ }_{S} V$ dans $V \times{ }_{S} V^{\vee} \times{ }_{S} V$ qui envoie $\left(w, v_{1}, v_{2}\right)$ sur $\left(v_{1}, w, v_{2}\right)$. On déduit de l'isomorphisme du lemme 3.4 et du théorème de changement de base propre un isomorphisme

$$
\nu^{*}(\Psi \otimes \Psi) \cong q_{!}^{\prime} \nu^{\prime *} \sigma^{*} \Psi[1]
$$

Comme $\sigma \circ \nu^{\prime}$ se factorise en

$$
\mathcal{V}^{\vee} \times_{S}\left[\left(V \times \times_{S} V\right) / \mathbb{G}_{\mathrm{m}, S}\right] \stackrel{\mathrm{Id} \times s}{\longrightarrow} \mathcal{V} \times_{S} \mathcal{V}^{\vee} \stackrel{\mu}{\longrightarrow} \mathcal{A}_{S}
$$

où $s:\left[\left(V \times{ }_{S} V\right) / \mathbb{G}_{\mathrm{m}, S}\right] \rightarrow \mathcal{V}$ est induit par le morphisme $V \times_{S} V \rightarrow V$ qui envoie $\left(v_{1}, v_{2}\right)$ sur $v_{1}-v_{2}$, que $\operatorname{pr}_{13} \circ q^{\prime}$ se factorise en

$$
\mathcal{V}^{\vee} \times_{S}\left[\left(V \times_{S} V\right) / \mathbb{G}_{\mathrm{m}, S}\right] \stackrel{\mathrm{pr}_{2}}{\longrightarrow}\left[\left(V \times_{S} V\right) / \mathbb{G}_{\mathrm{m}, S}\right] \longrightarrow \nu \times_{S} \nu
$$

et que $\Delta$ se factorise en

$$
\nu \stackrel{\Delta^{\prime}}{\longrightarrow}\left[\left(V \times{ }_{S} V\right) / \mathbb{G}_{\mathrm{m}, S}\right] \longrightarrow \mathcal{V} \times_{S} \mathcal{\nu}
$$

où $\Delta^{\prime}$ est induit par le morphisme diagonal $V \rightarrow V \times{ }_{S} V$, il suffit de construire un isomorphisme

$$
\operatorname{pr}_{2, !}(\operatorname{Id} \times s)^{*} \mu^{*} \Psi[1] \cong \Delta_{!}^{\prime} \overline{\mathbb{Q}}_{\ell}[2-2 r](-r) .
$$

BULLETIN DE LA SOCIÉTÉ MATHÉMATIQUE DE FRANCE 
Or, on a un diagramme à carrés cartésiens

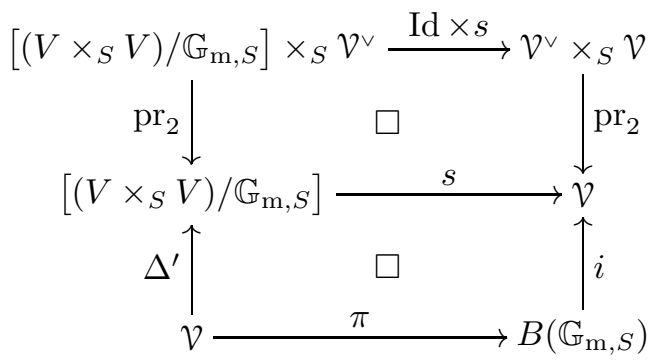

où $i$ est induit par la section nulle $S \rightarrow V$. Par suite, en utilisant de nouveau le théorème de changement de base propre, on voit qu'il suffit de construire un isomorphisme

$$
\operatorname{pr}_{2, !} \mu^{*} \Psi[1] \cong i_{!} \overline{\mathbb{Q}}_{\ell}[2-2 r](-r)
$$

Comme $i$ est représentable et une immersion fermée, on obtient un tel isomorphisme si l'on montre que $\operatorname{pr}_{2, !} \mu^{*} \Psi[1]$ est supporté par l'image de $i$ et que, après changement de base par $i$, on a un tel isomorphisme.

Or, pour tout point géométrique $s$ de $S$ et tout $v \in \mathcal{V}_{s}, v \neq 0$, induisant un morphisme $\langle v\rangle: \mathcal{V}_{s}^{\vee} \rightarrow \mathcal{A}_{s}, w \mapsto\langle w, v\rangle$, on a

$$
R \Gamma_{\mathrm{c}}\left(\mathcal{V}_{s}^{\vee},\langle v\rangle^{*} \Psi\right) \cong R \Gamma_{\mathrm{c}}\left(\mathcal{A}_{s},\langle v\rangle_{!}\langle v\rangle^{*} \Psi\right) \cong R \Gamma_{\mathrm{c}}\left(\mathcal{A}_{s}, \Psi\right)[2-2 r](1-r)=(0)
$$

d'après le lemme 3.3. Il reste donc à voir, d'après l'assertion (ii) de 1.4, que

$$
\operatorname{pr}_{2, !} \tilde{\mu}^{*} g_{!} \overline{\mathbb{Q}}_{\ell}[2]\left(\cong \operatorname{pr}_{2, !} \tilde{\mu}^{*} \alpha^{*} \beta_{*} \overline{\mathbb{Q}}_{\ell}[1]\right) \cong \overline{\mathbb{Q}}_{\ell}[2-2 r](-r)
$$

où $\widetilde{\mu}: \mathcal{V}^{\vee} \times_{S} B\left(\mathbb{G}_{\mathrm{m}, S}\right) \rightarrow B\left(\mathbb{G}_{\mathrm{m}, S}\right)$ envoie $\left(\mathcal{M} \rightarrow V^{\vee}, \mathcal{L}\right)$ sur $\mathcal{M} \otimes \mathcal{L}$. Par le théorème de changement de base propre, il revient de même de montrer que

$$
\left(\pi^{\vee}\right) ! \overline{\mathbb{Q}}_{\ell}[2] \cong \overline{\mathbb{Q}}_{\ell}[2-2 r](-r)
$$

c'est-à-dire

$$
\left(\pi^{\vee}\right) ! \overline{\mathbb{Q}}_{\ell} \cong \overline{\mathbb{Q}}_{\ell}[-2 r](-r),
$$

ce qui est clair puisque $\pi^{\vee}: \mathcal{V}^{\vee} \rightarrow B\left(\mathbb{G}_{\mathrm{m}, S}\right)$ est un fibré vectoriel de rang $r$.

\section{Commutation à la dualité et $t$-exactitude de la transformation de Fourier homogène}

THÉORÈME 4.1. - Il existe un isomorphisme canonique entre les foncteurs

$$
K \longmapsto \operatorname{Four}_{\mathcal{V} / S}\left(D_{\mathcal{V}}(K)\right) \text { et } K \longmapsto D_{\mathcal{V} \vee}\left(\operatorname{Four}_{\mathcal{V} / S}(K)\right)(-r)
$$

de $D_{\mathrm{c}}^{\mathrm{b}}\left(\mathcal{V}, \overline{\mathbb{Q}}_{\ell}\right)^{\text {opp }}$ dans $D_{\mathrm{c}}^{\mathrm{b}}\left(\mathcal{V}^{\vee}, \overline{\mathbb{Q}}_{\ell}\right)$. 
Démonstration. - On reprend mot pour mot un argument de Verdier pour la transformation de Fourier-Deligne. Par la dualité de Grothendieck, le foncteur

$$
D_{\mathcal{V} \vee} \circ \operatorname{Four}_{\mathcal{V} / S} \circ D_{\mathcal{V}}: D_{\mathrm{c}}^{\mathrm{b}}\left(\mathcal{V}, \overline{\mathbb{Q}}_{\ell}\right) \longrightarrow D_{\mathrm{c}}^{\mathrm{b}}\left(\mathcal{V}^{\vee}, \overline{\mathbb{Q}}_{\ell}\right)
$$

est isomorphe au foncteur $\mathcal{F}_{*}$ défini par

$$
\mathcal{F}_{*}(K)=\operatorname{pr}_{*}^{\vee}\left(\operatorname{pr}^{!} K \widetilde{\otimes} \mu^{!} \Psi^{\prime}\right)[1-r]
$$

où $\Psi^{\prime}=D_{\mathcal{A}}(\Psi)=\beta_{!} \overline{\mathbb{Q}}_{\ell}$.

Or on a un isomorphisme canonique bi-fonctoriel en $K$ dans $D_{\mathrm{c}}^{\mathrm{b}}\left(\mathcal{V}, \overline{\mathbb{Q}}_{\ell}\right)$ et $L$ dans $D_{\mathrm{c}}^{\mathrm{b}}\left(\mathcal{V}^{\vee}, \overline{\mathbb{Q}}_{\ell}\right)^{\text {opp }}$,

$$
\operatorname{Hom}_{D_{\mathrm{c}}^{\mathrm{b}}\left(\mathcal{V}, \overline{\mathbb{Q}}_{\ell}\right)}\left(\operatorname{Four}_{\mathcal{V} \vee} / S(L), K\right)=\operatorname{Hom}_{D_{\mathrm{c}}^{\mathrm{b}}\left(\mathcal{V} \vee, \overline{\mathbb{Q}}_{\ell}\right)}\left(L, \mathcal{F}_{*}(K)\right)
$$

puisque $\mathrm{pr}_{\text {! }}$ est l'adjoint à gauche de pr! et que l'on a l'isomorphisme de Grothendieck « cher à Cartan »

$$
\begin{aligned}
\operatorname{Hom}_{D_{\mathrm{c}}^{\mathrm{b}}\left(X, \overline{\mathbb{Q}}_{\ell}\right)}(K \otimes L, M) & \cong \operatorname{Hom}_{D_{\mathrm{c}}^{\mathrm{b}}\left(X, \overline{\mathbb{Q}}_{\ell}\right)}(K, \mathcal{H o m}(L, M)) \\
& \cong \operatorname{Hom}_{D_{\mathrm{c}}^{\mathrm{b}}\left(X, \overline{\mathbb{Q}}_{\ell}\right)}\left(K, D_{X}(L) \widetilde{\otimes} M\right)
\end{aligned}
$$

pour tout $S$-champ algébrique de type fini et tous $K, L, M \in \mathrm{ob} D_{\mathrm{c}}^{\mathrm{b}}\left(\mathcal{X}, \overline{\mathbb{Q}}_{\ell}\right)$.

Par suite, $\mathcal{F}_{*}$ est un adjoint à droite du foncteur Four $v^{\vee} / S$. Mais, il en est de même du foncteur $K \rightarrow \operatorname{Four}_{\mathcal{V} / S}(K)(r)$ qui est un quasi-inverse de $\operatorname{Four}_{\mathcal{v} v / S}$. Ces deux adjoints à droite sont donc quasi-isomorphes et le théorème est démontré.

ThÉORÈME 4.2. - La transformation de Fourier homogène Fourv/S est $t$ exacte.

Démonstration. - Compte tenu du théorème 4.1, il suffit de démontrer que, pour tout $\overline{\mathbb{Q}}_{\ell}$-faisceau pervers $K$ sur $\mathcal{V}, \operatorname{Four}_{\mathcal{V} / S}(K)$ est dans ${ }^{\mathrm{p}} D_{\overline{\mathrm{c}}}^{\geq 0}\left(\mathcal{V}^{\vee}, \overline{\mathbb{Q}}_{\ell}\right)$, c'est-à-dire que les faisceaux de cohomologie perverse ${ }^{\mathrm{p}} \mathcal{H}^{i}\left(\operatorname{Four}_{\mathcal{V} / S}(K)\right)$ sont tous nuls pour $i<0$.

Posons $L=\operatorname{pr}^{*} K \otimes \mu^{*} \Psi$ et démontrons dans un premier temps que $L$ est dans ${ }^{\mathrm{P}} D_{\overline{\mathrm{c}}}^{\geq 0}\left(\mathcal{V}^{\vee} \times{ }_{S} \mathcal{V}, \overline{\mathbb{Q}}_{\ell}\right)$. On a le dévissage

$$
\overline{\mathbb{Q}}_{\ell} \longrightarrow \beta_{*} \overline{\mathbb{Q}}_{\ell} \longrightarrow \alpha_{*} \overline{\mathbb{Q}}_{\ell}[-1](-1) \longrightarrow
$$

où on rappelle que $\alpha: B\left(\mathbb{G}_{\mathrm{m}, S}\right) \hookrightarrow \mathcal{A}_{S}$ est l'immersion fermée complémentaire de l'immersion ouverte $\beta: S \hookrightarrow \mathcal{A}$, et on a donc aussi le dévissage

$$
\operatorname{pr}^{*} K[r-1] \longrightarrow L \longrightarrow i_{*} i^{*}\left(\mathrm{pr}^{*} K[r-1]\right)[-1](-1) \longrightarrow
$$

où $i: \mathcal{H} \hookrightarrow \mathcal{V}^{\vee} \times{ }_{S} \mathcal{V}$ est l'immersion fermée induite par l'inclusion $\{\langle w, v\rangle=0\}$ $\subset V^{\vee} \times{ }_{S} V$. Maintenant, comme $K$ est pervers et que pr est lisse purement de dimension relative $r-1, \operatorname{pr}^{*} K[r-1]$ est pervers et $i_{*} i^{*}\left(\operatorname{pr}^{*} K[r-1]\right)$ est dans ${ }^{\mathrm{p}} D_{\mathrm{c}}^{[-1,0]}\left(\mathcal{V}^{\vee} \times{ }_{S} \mathcal{V}, \overline{\mathbb{Q}}_{\ell}\right)$ d'après [2], 4.1 .10 (ii). Par suite, $L$ est bien dans ${ }^{\mathrm{p}} D_{\overline{\mathrm{c}}}^{\geq 0}\left(\mathcal{V}^{\vee} \times{ }_{S} \mathcal{V}, \overline{\mathbb{Q}}_{\ell}\right)$. 
Pour terminer la démonstration, étudions le complexe $\pi ! \rho^{*} L[1]$ où on a noté encore $\pi: \mathcal{V}^{\vee} \times{ }_{S} V \rightarrow \mathcal{V}^{\vee}$ et $\rho: \mathcal{V}^{\vee} \times{ }_{S} V \rightarrow \mathcal{V}^{\vee} \times{ }_{S} \mathcal{V}$ les changements de base par $\bar{\pi}^{\vee}: \mathcal{V}^{\vee} \rightarrow S$ des morphismes $\pi: V \rightarrow S$ et $\rho: V \rightarrow \mathcal{V}$. Comme $\rho$ est lisse purement de dimension relative $1, \rho^{*} L[1]$ est dans ${ }^{\mathrm{p}} D_{\mathrm{c}}^{\geq 0}\left(\mathcal{V}^{\vee} \times{ }_{S} V, \overline{\mathbb{Q}}_{\ell}\right)$ et, comme $\pi$ est affine, $\pi_{!} \rho^{*} L[1]$ est donc dans ${ }^{\mathrm{p}} D_{\overline{\mathrm{c}}}^{\geq 0}\left(\mathcal{V}^{\vee}, \overline{\mathbb{Q}}_{\ell}\right)$ d'après [2], (4.1.2). De plus, on a $\pi_{!} \rho^{*} L[1]=\operatorname{pr}_{!}\left(L \otimes \rho_{!} \overline{\mathbb{Q}}_{\ell}[1]\right)$ d'après la formule des projections et on a un triangle distingué

$$
\overline{\mathbb{Q}}_{\ell} \longrightarrow \rho_{!} \overline{\mathbb{Q}}_{\ell}[1] \longrightarrow \overline{\mathbb{Q}}_{\ell}[-1](-1) \longrightarrow .
$$

Par conséquent, on a un triangle distingué

$$
\operatorname{pr}_{!}^{\vee} L \longrightarrow \pi ! \rho^{*} L[1] \longrightarrow \operatorname{pr}_{!}^{\vee} L[-1](-1) \longrightarrow
$$

où $\pi ! \rho^{*} L[1]$ est dans ${ }^{\mathrm{p}} D_{\overline{\mathrm{c}}}^{\geq 0}\left(\mathcal{V}^{\vee}, \overline{\mathbb{Q}}_{\ell}\right)$. En prenant la suite exacte longue de cohomologie perverse, on en déduit des isomorphismes

$$
\mathrm{p} \mathcal{H}^{i-2} \operatorname{pr}_{!}^{\vee} L(-1) \stackrel{\sim}{\longrightarrow} \mathrm{p}^{i} \operatorname{pr}_{!}^{\vee} L, \quad \forall i<0 .
$$

Par suite, si l'un des faisceaux de cohomologie perverse ${ }^{\mathrm{p}} \mathcal{H}^{i}$ de $\operatorname{pr}_{!}^{\vee} L=$ $\operatorname{Four}_{\mathcal{V} / S}(K)$ avec $i<0$ était non nul, il en serait de même de tous les ${ }^{\mathrm{p}} \mathcal{H}^{i-2 n}$ avec $n \geq 0$, ce qui contredit le fait que $\operatorname{Four}_{\mathcal{V} / S}(K)$ est un complexe borné (cf. corollaire 1.9), d'où la conclusion.

\section{Compléments}

5.1. Supposons tout d'abord que $S=\operatorname{Spec}\left(\mathbb{F}_{q}\right)$ pour un corps fini $\mathbb{F}_{q}$ à $q$ éléments et calculons l'action de la transformation de Fourier homogène sur les fonctions «trace de Frobenius ».

On note $\left|\mathcal{V}\left(\mathbb{F}_{q}\right)\right|$ l'ensemble des classes d'isomorphie des objets de la catégorie $\mathcal{V}\left(\mathbb{F}_{q}\right)$; on a

$$
\left|\mathcal{V}\left(\mathbb{F}_{q}\right)\right|=\{0\} \cup \mathbb{P}(V)\left(\mathbb{F}_{q}\right) .
$$

Pour tout $K \in D_{\mathrm{c}}^{\mathrm{b}}\left(\mathcal{V}, \overline{\mathbb{Q}}_{\ell}\right)$, on note

$$
\tau_{K}:\left|\mathcal{V}\left(\mathbb{F}_{q}\right)\right| \longrightarrow \overline{\mathbb{Q}}_{\ell}
$$

la fonction qui associe à $v \in\left|\mathcal{V}\left(\mathbb{F}_{q}\right)\right|$ la trace de l'action de l'endomorphisme de Frobenius géométrique $\operatorname{Frob}_{q}$ relatif à $\mathbb{F}_{q}$ sur la fibre de $K$ en n'importe quel point géométrique de $\mathcal{V}$ localisé en $v$. Bien entendu on pose la même définition pour $\mathcal{V}^{\vee}$.

Lemme 5.2. - Pour tout $K \in D_{\mathrm{c}}^{\mathrm{b}}\left(\mathcal{V}, \overline{\mathbb{Q}}_{\ell}\right)$ et tout $w \in\left|\mathcal{V}^{\vee}\left(\mathbb{F}_{q}\right)\right|$, on a

$$
\tau_{\text {Four }_{\mathcal{V} / S}(K)}(w)=\tau_{K}(0)-\sum_{v \in \mathbb{P}(V)\left(\mathbb{F}_{q}\right)} \tau_{K}(v)+q \sum_{\substack{v \in \mathbb{P}(V)\left(\mathbb{F}_{q}\right) \\(w, v) \in H\left(\mathbb{F}_{q}\right)}} \tau_{K}(v)
$$

où on rappelle que $H \subset \mathbb{P}\left(V^{\vee}\right) \times_{S} \mathbb{P}(V)$ est l'hypersurface d'incidence. 
Démonstration. - On applique la formule des traces de Grothendieck, soit directement, soit à travers la compatibilité de la transformation de Fourier homogène à celle de Fourier-Deligne prouvée dans la section 2 .

5.3. On revient à la situation générale et on considère la sous-catégorie pleine

$$
D_{\mathrm{c}, \dagger}^{\mathrm{b}}\left(\mathbb{P}(V), \overline{\mathbb{Q}}_{\ell}\right) \subset D_{\mathrm{c}}^{\mathrm{b}}\left(\mathbb{P}(V), \overline{\mathbb{Q}}_{\ell}\right)
$$

définie par la condition $\left(\bar{\pi}^{\circ}\right) ! K=\left(\bar{\pi}^{\circ}\right)_{*} K=(0)$, où on rappelle que le morphisme projectif $\bar{\pi}^{\circ}: \mathcal{V}^{\circ}=\mathbb{P}(V) \rightarrow S$ est la projection canonique. Cette souscatégorie est triangulée et est sa propre image par la dualité $D_{\mathbb{P}(V)}$.

La proposition suivante est une variante de résultats de Brylinski sur la transformation de Radon géométrique (cf. [3]).

Proposition 5.4. - (i) Pour tout $K \in$ ob $D_{\mathrm{c}}^{\mathrm{b}}\left(\mathbb{P}(V), \overline{\mathbb{Q}}_{\ell}\right)$, on $a\left(\bar{\pi}^{\circ}\right)_{*} K=(0)$ si et seulement si la flèche d'oubli des supports

$$
j_{!} K \longrightarrow j_{*} K
$$

est un isomorphisme dans $D_{\mathrm{c}}^{\mathrm{b}}\left(\mathcal{V}, \overline{\mathbb{Q}}_{\ell}\right)$.

(ii) Pour tout $K \in \mathrm{ob} D_{\mathrm{c}}^{\mathrm{b}}\left(\mathbb{P}(V), \overline{\mathbb{Q}}_{\ell}\right)$, on $a\left(\bar{\pi}^{\circ}\right)_{*} K=(0)$ si et seulement si $\left(\bar{\pi}^{\vee \circ}\right)_{*} \operatorname{Rad}_{\mathbb{P}(V) / S}(K)=(0)$, et la transformation de Radon géométrique $\operatorname{Rad}_{\mathbb{P}(V) / S}$ induit une équivalence de catégories triangulées

$$
\operatorname{Rad}_{\mathbb{P}\left(V^{\vee}\right) / S, \uparrow}: D_{\mathrm{c}, \dagger}^{\mathrm{b}}\left(\mathbb{P}(V), \overline{\mathbb{Q}}_{\ell}\right) \stackrel{\sim}{\longrightarrow} D_{\mathrm{c}, \dagger}^{\mathrm{b}}\left(\mathbb{P}\left(V^{\vee}\right), \overline{\mathbb{Q}}_{\ell}\right),
$$

de quasi-inverse induit par $\operatorname{Rad}_{\mathbb{P}\left(V^{\vee}\right) / S}(r-2)$.

Nous utiliserons le lemme d'homotopie bien connu suivant (cf. [12] par exemple) :

Lemme 5.5. - Soit $f: X \rightarrow S$ un $S$-schéma séparé et de type fini, muni d'une $S$-action contractante de $\mathbb{G}_{\mathrm{m}, S}$, i.e. d'un morphisme $\mathbb{A}_{S}^{1} \times_{S} X \rightarrow X$, $(u, x) \mapsto u \cdot x$ tel que $1 \cdot x=x$ et $(t u) \cdot x=t \cdot(u \cdot x)$ quels que soient $x \in X$, $t \in \mathbb{G}_{\mathrm{m}, S}$ et $u \in \mathbb{A}_{S}^{1}$. Notons $\rho: X \rightarrow X$ le champ quotient de $X$ par cette action de $\mathbb{G}_{\mathrm{m}, S}$. Alors, pour tout $K \in \mathrm{ob} D_{\mathrm{c}}^{\mathrm{b}}\left(X, \overline{\mathbb{Q}}_{\ell}\right)$ la flèche de restriction

$$
f_{*} \rho^{*} K \longrightarrow g_{*}\left(\rho^{*} K \mid Y\right)
$$

au fermé

$$
g: Y=\left\{x \in X \mid t \cdot x=x, \forall t \in \mathbb{G}_{\mathrm{m}, S}\right\} \longrightarrow S
$$

des points fixes de cette action, est un isomorphisme.

Démonstration. - Par dévissage de $K$ en un complexe nul sur $y=\left[Y / \mathbb{G}_{\mathrm{m}, S}\right] \subset$ $X$ et un complexe supporté sur $y$, on peut supposer que la restriction de $K$ à $y$ est nulle, et il s'agit alors de démontrer que $f_{*} \rho^{*} K=(0)$.

Soit $\tau: \mathbb{A}_{S}^{1} \times_{S} X \rightarrow \mathbb{A}_{S}^{1} \times_{S} X$ l'endomorphisme défini par $\tau(u, x)=(u, u \cdot x)$. On a un isomorphisme évident entre les restrictions des complexes $\operatorname{pr}_{X}^{*} \rho^{*} K$ et $\tau^{*} \operatorname{pr}_{X}^{*} \rho^{*} K$ à l'ouvert $\mathbb{G}_{\mathrm{m}, S} \times_{S} X \subset \mathbb{A}_{S}^{1} \times_{S} X$, et comme la restriction de $K$ à 
$y$ est nulle par hypothèse, il en est de même de la restriction de $\tau^{*} \operatorname{pr}_{X}^{*} \rho^{*} K$ au fermé $X \hookrightarrow \mathbb{A}_{S}^{1} \times_{S} X, x \mapsto(0, x)$. On en déduit l'existence d'une flèche

$$
\tau^{*} \operatorname{pr}_{X}^{*} \rho^{*} K \longrightarrow \operatorname{pr}_{X}^{*} \rho^{*} K
$$

sur $\mathbb{A}_{S}^{1} \times_{S} X$ tout entier dont la restriction à $\mathbb{G}_{\mathrm{m}, S} \times_{S} X$ est un isomorphisme. Par adjonction, cette flèche nous donne une flèche

$$
\operatorname{pr}_{X}^{*} \rho^{*} K \longrightarrow \tau_{*} \operatorname{pr}_{X}^{*} \rho^{*} K
$$

et donc une flèche

$$
\left(\operatorname{pr}_{\mathbb{A}_{S}^{1}}\right)_{*} \operatorname{pr}_{X}^{*} \rho^{*} K \longrightarrow\left(\operatorname{pr}_{\mathbb{A}_{S}^{1}}\right) * \operatorname{pr}_{X}^{*} \rho^{*} K
$$

puisque $\operatorname{pr}_{\mathbb{A}_{S}^{1}} \circ \tau=\operatorname{pr}_{\mathbb{A}_{S}^{1}}$.

D'après la formule de Künneth, le complexe $\left(\operatorname{pr}_{\mathbb{A}_{S}^{1}}\right) * \operatorname{pr}_{X}^{*} \rho^{*} K$ est l'image réciproque du complexe $f_{*} \rho^{*} K$ par la projection canonique $\mathbb{A}_{S}^{1} \rightarrow S$ et la formation de la flèche $\left(^{*}\right)$ commute à la restriction à toute section $S \rightarrow \mathbb{A}_{S}^{1}$ de cette même projection canonique. Il ne reste plus qu'à remarquer que la restriction à la section $1: S \rightarrow \mathbb{A}_{S}^{1}$ de $\left(^{*}\right)$ est l'identité alors que celle à la section $0: S \rightarrow \mathbb{A}_{S}^{1}$ est nulle pour conclure que $f_{*} \rho^{*} K=(0)$.

Démonstration de la proposition 5.4. - Notons encore $j$ l'inclusion de $V^{\circ}$ dans $V$ et $\rho^{\circ}: V^{\circ} \rightarrow \mathbb{P}(V)$ le morphisme quotient. La flèche d'oubli des supports de l'énoncé est un isomorphisme si et seulement si la flèche d'oubli des supports

$$
j_{!} \rho^{*} K \longrightarrow j_{*} \rho^{*} K
$$

est un isomorphisme dans $D_{\mathrm{c}}^{\mathrm{b}}\left(V, \overline{\mathbb{Q}}_{\ell}\right)$. Le lemme d'homotopie précédent appliqué à $j_{*} K \in \mathrm{ob} D_{\mathrm{c}}^{\mathrm{b}}\left(\mathcal{V}, \overline{\mathbb{Q}}_{\ell}\right)$ assure que cette dernière flèche d'oubli des supports est un isomorphisme si et seulement si $\pi_{*}^{\circ} \rho^{*} K=\pi_{*} j_{*} \rho^{*} K=(0)$ où $\pi^{\circ}=\pi \circ j$ : $V^{\circ} \rightarrow S$. On a

$$
\pi_{*}^{\circ} \rho^{*} K=\left(\bar{\pi}^{\circ}\right)_{*}\left(K \otimes \rho_{*} \overline{\mathbb{Q}}_{\ell}\right)
$$

d'après la formule de Künneth, puisque $\rho$ est un $\mathbb{G}_{\mathrm{m}, S}$-torseur, d'où un triangle distingué

$$
\left(\bar{\pi}^{\circ}\right)_{*} K \longrightarrow \pi_{*}^{\circ} \rho^{*} K \longrightarrow\left(\bar{\pi}^{\circ}\right)_{*} K[-1](-1) \longrightarrow .
$$

Par suite, $\pi_{*}^{\circ} \rho^{*} K=(0)$ si et seulement si la flèche de co-bord

$$
\left(\bar{\pi}^{\circ}\right)_{*} K[-1](-1) \longrightarrow\left(\bar{\pi}^{\circ}\right)_{*} K[1]
$$

est un isomorphisme, c'est-à-dire si et seulement si $\left(\bar{\pi}^{\circ}\right)_{*} K=(0)$ puisque ce dernier complexe est borné, d'où l'assertion (i) de la proposition. 
Passons à la démonstration de l'assertion (ii). On a

$$
\begin{aligned}
\left(\bar{\pi}^{\vee \circ}\right)_{*} \operatorname{Rad}_{\mathbb{P}(V) / S}(K) & =\left(\bar{\pi}^{\vee \circ}\right)_{*}\left(q^{\vee}\right)_{*} q^{*} K[r-2] \\
& =\left(\bar{\pi}^{\circ}\right)_{*} q_{*} q^{*} K[r-2] \\
& =\left(\bar{\pi}^{\circ}\right)_{*}\left(K \otimes q_{*} \overline{\mathbb{Q}}_{\ell}[r-2]\right) \\
& =\bigoplus_{i=0}^{r-2}\left(\bar{\pi}^{\circ}\right)_{*} K[r-2-2 i](-i),
\end{aligned}
$$

et donc $\left(\bar{\pi}^{\vee 0}\right)_{*} \operatorname{Rad}_{\mathbb{P}(V) / S}(K)=(0)$ si et seulement si $\left(\bar{\pi}^{\circ}\right)_{*} K=(0)$.

Si $\left(\bar{\pi}^{\circ}\right)_{*} K=(0)$, on a

$$
\left(j^{\vee}\right)^{*} \operatorname{Four}_{\mathcal{V} / S}\left(j_{!} K\right) \cong \operatorname{Rad}_{\mathbb{P}(V) / S}(K)(-1)
$$

d'après la proposition 1.6 , et donc

$$
j^{*} \operatorname{Four}_{\mathcal{v}} / S\left(\left(j^{\vee}\right) !\left(j^{\vee}\right)^{*} \operatorname{Four}_{\mathcal{V} / S}(j ! K)\right) \cong \operatorname{Rad}_{\mathbb{P}\left(V^{\vee}\right) / S}\left(\operatorname{Rad}_{\mathbb{P}(V) / S}(K)\right)(-2)
$$

d'après ce qui précède et encore la proposition 1.6. Or on a le triangle distingué

$$
\begin{aligned}
j^{*} \operatorname{Four}_{\mathcal{v} / S}\left(\left(j^{\vee}\right)_{!}\left(j^{\vee}\right)^{*} \operatorname{Four}_{\mathcal{V} / S}(j ! K)\right) \longrightarrow j^{*} \operatorname{Four}_{\mathcal{V} \vee / S}\left(\operatorname{Four}_{\mathcal{V} / S}(j ! K)\right) \\
\longrightarrow j^{*} \operatorname{Four}_{\mathcal{V} \vee / S}\left(\left(i^{\vee}\right)_{*}\left(i^{\vee}\right)^{*} \operatorname{Four}_{\mathcal{V} / S}(j ! K)\right) \longrightarrow,
\end{aligned}
$$

et on a

$$
j^{*} \operatorname{Four}_{\mathcal{V} \vee / S}\left(\operatorname{Four}_{\mathcal{V} / S}(j ! K)\right) \cong K(-r)
$$

d'après le théorème 3.1 , et

$$
\begin{aligned}
j^{*} \operatorname{Four}_{\mathcal{V} \vee / S}\left(\left(i^{\vee}\right)_{*}\left(i^{\vee}\right)^{*} \operatorname{Four}_{\mathcal{V} / S}(j ! K)\right) & =j^{*} \pi^{*} \sigma^{*}\left(i^{\vee}\right)^{*} \operatorname{Four}_{\mathcal{V} / S}\left(j_{!} K\right)[r] \\
& =j^{*} \pi^{*} \sigma^{*} \sigma^{*} \pi ! j ! K[2 r] \\
& =j^{*} \pi^{*} \pi ! j ! K[2 r]
\end{aligned}
$$

d'après des cas particuliers du lemme 1.7 et la proposition 1.8. Comme le morphisme déduit de $\pi \circ j: \mathbb{P}(V) \rightarrow B\left(\mathbb{G}_{\mathrm{m}, S}\right)$ par le changement de base $S \rightarrow B\left(\mathbb{G}_{\mathrm{m}, S}\right)$ est le morphisme structural $\pi^{\circ}: V^{\circ} \rightarrow S$ qui se factorise en le morphisme quotient $\rho^{\circ}: V^{\circ} \rightarrow \mathbb{P}(V)$ et $\bar{\pi}^{\circ}: \mathbb{P}(V) \rightarrow S$, il résulte de l'hypothèse $\left(\bar{\pi}^{\circ}\right)_{*} K=(0)$ que $\pi_{!} j_{!} K=(0)$, et donc que

$$
\operatorname{Rad}_{\mathbb{P}\left(V^{\vee}\right) / S}\left(\operatorname{Rad}_{\mathbb{P}(V) / S}(K)\right) \cong K(2-r),
$$

et l'assertion (ii) est démontrée.

Lemme 5.6. - (i) La sous catégorie strictement pleine $\mathcal{S}$ de $\operatorname{Perv}\left(\mathbb{P}(V), \overline{\mathbb{Q}}_{\ell}\right)$ définie comme l'image essentielle du foncteur exact

$$
\left(\bar{\pi}^{\circ}\right)^{*}(\cdot)[r-1]: \operatorname{Perv}\left(S, \overline{\mathbb{Q}}_{\ell}\right) \longrightarrow \operatorname{Perv}\left(\mathbb{P}(V), \overline{\mathbb{Q}}_{\ell}\right),
$$

est une sous-catégorie de Serre, c'est-à-dire est stable par sous-quotients et par extensions.

BULLETIN DE LA SOCIÉTÉ MATHÉMATIQUE DE FRANCE 
En particulier, tout $\overline{\mathbb{Q}}_{\ell}$-faisceau pervers $K$ sur $\mathbb{P}(V)$ admet un plus grand sous-faisceau pervers (resp. faisceau pervers quotient) qui provient de $S$ et qui n'est autre que

$$
\left(\bar{\pi}^{\circ}\right)^{*}\left(\mathrm{p}^{\mathrm{H}}{ }^{1-r}\left(\bar{\pi}^{\circ}\right)_{*} K\right)[r-1] \hookrightarrow K
$$

(resp. $\left.K \rightarrow\left(\bar{\pi}^{\circ}\right) !\left(\mathrm{p} \mathcal{H}^{r-1}\left(\bar{\pi}^{\circ}\right)_{*} K\right)[1-r]=\left(\bar{\pi}^{\circ}\right)^{*}\left({ }^{\mathrm{P}} \mathcal{H}^{r-1}\left(\bar{\pi}^{\circ}\right)_{*} K\right)[r-1](r-1)\right)$

où l'injection (resp. la surjection) est induite par la flèche d'adjonction $\left(\bar{\pi}^{\circ}\right)^{*}\left(\bar{\pi}^{\circ}\right)_{*} \rightarrow$ Id $\left(\right.$ resp. Id $\left.\rightarrow\left(\bar{\pi}^{\circ}\right)^{!}\left(\bar{\pi}^{\circ}\right)_{*}\right)$.

(ii) Notons

$$
\mathcal{P}\left(\mathbb{P}(V), \overline{\mathbb{Q}}_{\ell}\right)=\operatorname{Perv}\left(\mathbb{P}(V), \overline{\mathbb{Q}}_{\ell}\right) / \mathcal{S}
$$

la catégorie abélienne quotient au sens de Serre (cf. [7], chap. III), obtenue en inversant dans la catégorie abélienne $\operatorname{Perv}\left(\mathbb{P}(V), \overline{\mathbb{Q}}_{\ell}\right)$ les morphismes dont les noyau et conoyau sont dans $\mathcal{S}$. Alors les objets simples de $\mathcal{P}\left(\mathbb{P}(V), \overline{\mathbb{Q}}_{\ell}\right)$ sont exactement les objets simples de $\operatorname{Perv}\left(\mathbb{P}(V), \overline{\mathbb{Q}}_{\ell}\right)$ qui ne sont pas dans $\mathcal{S}$.

(iii) Un $\overline{\mathbb{Q}}_{\ell}$-faisceau pervers $K$ sur $\mathbb{P}(V)$ est dans $\mathcal{S}$ si et seulement si $\left(\rho^{\circ}\right)^{*} K[1]$ est isomorphe dans $\operatorname{Perv}\left(V^{\circ}, \overline{\mathbb{Q}}_{\ell}\right)$ à $\left(\rho^{\circ}\right)^{*} K^{\prime}[1]$ pour un objet $K^{\prime}$ de $\mathcal{S}$, c'est-à-dire si et seulement si $\left(\rho^{\circ}\right)^{*} K[1]$ est isomorphe $\grave{a}\left(\pi^{\circ}\right)^{*} M^{\prime}[r]$ pour un objet $M^{\prime}$ de $\operatorname{Perv}\left(S, \overline{\mathbb{Q}}_{\ell}\right)$, et on a l'inclusion

$$
\left(\pi^{\circ}\right)^{*}\left(\operatorname{ob} \operatorname{Perv}\left(B\left(\mathbb{G}_{\mathrm{m}, S}\right), \overline{\mathbb{Q}}_{\ell}\right)\right)[r] \subset \mathrm{ob} \mathcal{S}
$$

où on a noté encore par $\pi^{\circ}: \mathbb{P}(V) \rightarrow B\left(\mathbb{G}_{\mathrm{m}, S}\right)$ le morphisme induit par le morphisme structural $\pi^{\circ}: V^{\circ} \rightarrow S$.

Démonstration. - Mis à part l'assertion de stabilité par extensions, la partie (i) du lemme est démontrée dans la section 4.2.6 de [2].

Pour tout couple $\left(M_{1}, M_{2}\right)$ de $\overline{\mathbb{Q}}_{\ell}$-faisceaux pervers sur $S$, le foncteur exact $\left(\bar{\pi}^{\circ}\right)^{*}(\cdot)[r-1]$ induit un isomorphisme

$$
\operatorname{Ext}^{1}\left(M_{1}, M_{2}\right) \stackrel{\sim}{\longrightarrow} \operatorname{Ext}^{1}\left(\left(\bar{\pi}^{\circ}\right)^{*} M_{1}[r-1],\left(\bar{\pi}^{\circ}\right)^{*} M_{2}[r-1]\right)
$$

puisque l'on a

$$
\begin{aligned}
\operatorname{Ext}^{1}\left(\left(\bar{\pi}^{\circ}\right)^{*} M_{1}[r-1],\left(\bar{\pi}^{\circ}\right)^{*} M_{2}[r-1]\right) & =\operatorname{Ext}^{1}\left(\left(\bar{\pi}^{\circ}\right)^{*} M_{1},\left(\bar{\pi}^{\circ}\right)^{*} M_{2}\right) \\
& =\operatorname{Ext}^{1}\left(M_{1},\left(\bar{\pi}^{\circ}\right)_{*}\left(\bar{\pi}^{\circ}\right)^{*} M_{2}\right) \\
& =\operatorname{Ext}^{1}\left(M_{1} M_{2} \otimes\left(\bar{\pi}^{\circ}\right)_{*} \overline{\mathbb{Q}}_{\ell}\right) \\
& =\operatorname{Ext}^{1}\left(M_{1}, \bigoplus_{i=0}^{r-1} M_{2}[-2 i](-i)\right) \\
& =\bigoplus_{i=0}^{r-1} \operatorname{Ext}^{1-2 i}\left(M_{1}, M_{2}(-i)\right) \\
& =\operatorname{Ext}^{1}\left(M_{1}, M_{2}\right) .
\end{aligned}
$$

Par suite, $\mathcal{S}$ est stable par extensions, ce qui achève la preuve de la partie (i) du lemme. 
La partie (ii) est formelle : elle vaut pour toute catégorie quotient au sens de Serre d'une catégorie abélienne nœethérienne et artinienne.

Enfin, la première assertion de la partie (iii) résulte de l'isomorphisme évident

$$
K \cong \mathrm{P} \mathcal{H}^{-1}\left(\rho_{*}^{\circ}\left(\rho^{\circ}\right)^{*} K[1]\right)
$$

et la seconde assertion résulte de la première puisque le morphisme déduit par le changement de base $S \rightarrow B\left(\mathbb{G}_{\mathrm{m}, S}\right)$ du morphisme $\pi^{\circ}: \mathbb{P}(V) \rightarrow B\left(\mathbb{G}_{\mathrm{m}, S}\right)$ est le morphisme structural $\pi^{\circ}: V^{\circ} \rightarrow S$ et se factorise donc en $\rho^{\circ}: V^{\circ} \rightarrow \mathbb{P}(V)$ et $\bar{\pi}^{\circ}: \mathbb{P}(V) \rightarrow S$.

On dira que les objets de $\mathcal{S}$ sont les $\overline{\mathbb{Q}}_{\ell}$-faisceaux pervers sur $\mathbb{P}(V)$ qui proviennent de $S$.

Bien entendu, on peut remplacer $V$ par $V^{\vee}$ dans ce qui précède et on obtient la sous-catégorie épaisse et stable par extensions $\mathcal{S}^{\vee}$ de $\operatorname{Perv}\left(\mathbb{P}\left(V^{\vee}\right), \overline{\mathbb{Q}}_{\ell}\right)$ dont les objets sont ceux qui proviennent de $S$, et la catégorie abélienne quotient au sens de Serre

$$
\mathcal{P}\left(\mathbb{P}\left(V^{\vee}\right), \overline{\mathbb{Q}}_{\ell}\right)=\operatorname{Perv}\left(\mathbb{P}\left(V^{\vee}\right), \overline{\mathbb{Q}}_{\ell}\right) / \mathcal{S}^{\vee} .
$$

Proposition 5.7 (Brylinski [3]). - (i) Si K est un $\overline{\mathbb{Q}}_{\ell}$-faisceau pervers sur $\mathbb{P}(V)$, alors, pour chaque entier $i \neq 0$, le $i$-ème faisceau de cohomologie perverse de $\operatorname{Rad}_{\mathbb{P}(V) / S}(K)$ provient de $S$, plus précisément est canoniquement isomorphe $\grave{a}$

$$
\mathrm{p}_{\mathcal{H}}{ }^{i} \operatorname{Rad}_{\mathbb{P}(V) / S}(K) \cong \begin{cases}\left(\bar{\pi}^{\vee \circ}\right)^{*}\left(\mathrm{p} \mathcal{H} \mathcal{H}^{i-1}\left(\bar{\pi}^{\circ}\right)_{*} K\right)[r-1] & \text { si } i<0, \\ \left(\bar{\pi}^{\vee \circ}\right)^{*}\left(\mathrm{p} \mathcal{H} \mathcal{H}^{i+1}\left(\bar{\pi}^{\circ}\right)_{*} K(1)\right)[r-1] & \text { si } i>0 .\end{cases}
$$

En particulier, $\operatorname{Rad}_{\mathbb{P}(V) / S}(K)$ est pervers si $\left(\bar{\pi}^{\circ}\right)_{*} K \in{ }^{\mathrm{p}} D_{\mathrm{c}}^{[-1,1]}\left(S, \overline{\mathbb{Q}}_{\ell}\right)$.

(ii) Le foncteur ${ }^{\mathrm{p}} \mathcal{H}^{0} \operatorname{Rad}_{\mathbb{P}(V) / S}: \operatorname{Perv}\left(\mathbb{P}(V), \overline{\mathbb{Q}}_{\ell}\right) \rightarrow \operatorname{Perv}\left(\mathbb{P}\left(V^{\vee}\right), \overline{\mathbb{Q}}_{\ell}\right)$ envoie la sous-catégorie $\mathcal{S}$ dans la sous-catégorie $\mathcal{S}^{\vee}$ et induit donc un foncteur noté $\mathcal{R}_{\mathbb{P}(V) / S}$ de la catégorie abélienne $\mathcal{P}\left(\mathbb{P}(V), \overline{\mathbb{Q}}_{\ell}\right)$ dans la catégorie abélienne $\mathcal{P}\left(\mathbb{P}\left(V^{\vee}\right), \overline{\mathbb{Q}}_{\ell}\right)$. Ce foncteur $\mathcal{R}_{\mathbb{P}(V) / S}$ est exact et est une équivalence de catégories abéliennes de quasi-inverse $\mathcal{R}_{\mathbb{P}\left(V^{\vee}\right) / S}(r-2)$.

Démonstration. - Si $K \in \operatorname{ob} \operatorname{Perv}\left(\mathbb{P}(V), \overline{\mathbb{Q}}_{\ell}\right)$, le complexe $\left(j^{\vee}\right)^{*} \operatorname{Four}_{\mathcal{V} / S}(j ! K)$ est dans ${ }^{\mathrm{p}} D_{\mathrm{c}}^{\leq 0}\left(\mathbb{P}\left(V^{\circ}\right), \overline{\mathbb{Q}}_{\ell}\right)$ puisque $j$ ! est $t$-exact à droite et que Four $\mathcal{V} / S$ est $t$ exact d'après le théorème 4.2. Le triangle distingué de la proposition 1.6 donne donc les isomorphismes cherchés pour $i>0$.

Dualement, on a le triangle distingué

$$
\operatorname{Rad}_{\mathbb{P}(V) / S}(K) \longrightarrow\left(j^{\vee}\right)^{*} \operatorname{Four}_{\mathcal{V} / S}\left(j_{*} K\right)(1) \longrightarrow\left(\bar{\pi}^{\vee \circ}\right)^{*}\left(\bar{\pi}^{\circ}\right)_{*} K[r-1] \longrightarrow
$$

et le complexe $\left(j^{\vee}\right)^{*} \operatorname{Four}_{\mathcal{V} / S}\left(j_{*} K\right)$ est dans ${ }^{\mathrm{p}} D_{\bar{c}}^{\geq 0}\left(\mathbb{P}\left(V^{\circ}\right), \overline{\mathbb{Q}}_{\ell}\right)$, d'où les isomorphismes cherchés pour $i<0$ et l'assertion (i) est démontrée. 
Le calcul

$$
\begin{aligned}
\operatorname{Rad}_{\mathbb{P}(V) / S}\left(\left(\bar{\pi}^{\circ}\right)^{*} M[r-1]\right) & =\left(q^{\vee}\right)_{*} q^{*}\left(\bar{\pi}^{\circ}\right)^{*} M[2 r-3] \\
& =\left(q^{\vee}\right)_{*}\left(q^{\vee}\right)^{*}\left(\bar{\pi}^{\vee \circ}\right)^{*} M[2 r-3] \\
& =\left(\bar{\pi}^{\vee \circ}\right)^{*} M \otimes\left(q^{\vee}\right)_{*} \overline{\mathbb{Q}}_{\ell}[2 r-3] \\
& =\bigoplus_{i=0}^{r-2}\left(\bar{\pi}^{\vee \circ}\right)^{*} M[2 r-3-2 i](-i)
\end{aligned}
$$

implique la formule

$\mathrm{p}_{\mathcal{H}} \mathcal{R a d}_{\mathbb{P}(V) / S}\left(\left(\bar{\pi}^{\circ}\right)^{*} M[r-1]\right)= \begin{cases}\left(\bar{\pi}^{\vee 0}\right)^{*} M[r-1]\left(-r^{\prime}\right) & \text { si } r-2=2 r^{\prime} \text { est pair, } \\ (0) & \text { si } r \text { est impair, }\end{cases}$ pour tout $\overline{\mathbb{Q}}_{\ell}$-faisceau pervers $M$ sur $S$, et donc l'inclusion

$$
{ }^{\mathrm{p}} \mathcal{H}^{0} \operatorname{Rad}_{\mathbb{P}(V) / S}(\mathrm{ob} \mathcal{S}) \subset \mathrm{ob} \mathcal{S}^{\vee} .
$$

Compte tenu du triangle distingué de la proposition 1.6 et de la partie (iii) du lemme 5.7, on a aussi l'inclusion

$$
{ }^{\mathrm{p}} \mathcal{H}^{0}\left(j^{\vee}\right)^{*} \operatorname{Four}_{V / S}\left(j_{!}(\mathrm{ob} \mathcal{S})\right) \subset \mathrm{obS}^{\vee}
$$

et le foncteur induit par ${ }^{\mathrm{p}} \mathcal{H}^{0} \circ\left(j^{\vee}\right)^{*} \circ \operatorname{Four}_{V / S} \circ j_{\text {! }}$ de $\mathcal{P}\left(\mathbb{P}(V), \overline{\mathbb{Q}}_{\ell}\right)$ dans $\mathcal{P}\left(\mathbb{P}\left(V^{\vee}\right), \overline{\mathbb{Q}}_{\ell}\right)$ n'est autre que $\mathcal{R}_{\mathbb{P}(V) / S}$. On conclut en raisonnant comme dans la démonstration de la partie (ii) de la proposition 5.4.

Corollaire 5.8 (Brylinski [3]). - (i) Si K est un $\overline{\mathbb{Q}}_{\ell}$-faisceau pervers irréductible sur $\mathbb{P}(V)$ qui ne provient pas de $S$, le faisceau pervers $L=$ $\mathrm{p}^{\mathcal{H}}{ }^{0} \operatorname{Rad}_{\mathbb{P}(V) / S}(K)$ sur $\mathbb{P}(V)$ admet une filtration à trois crans

$$
(0)=L_{0} \subset L_{1} \subset L_{2} \subset L_{3}=L
$$

telle que $L_{1}$ et $L_{3} / L_{2}$ proviennent de $S$ et que $L_{2} / L_{1}$ soit un $\overline{\mathbb{Q}}_{\ell}$-faisceau pervers irréductible sur $\mathbb{P}\left(V^{\vee}\right)$.

(ii) Si $K$ est un $\overline{\mathbb{Q}}_{\ell}$-faisceau pervers irréductible sur $\mathbb{P}(V)$ tel que $\left(\bar{\pi}^{\circ}\right)_{*} K=$ $(0)$, alors $\operatorname{Rad}_{\mathbb{P}(V) / S}(K)$ est un $\overline{\mathbb{Q}}_{\ell}$-faisceau pervers irréductible sur $\mathbb{P}\left(V^{\vee}\right)$.

Démonstration. - L'assertion (i) résulte de l'assertion (ii) de la proposition 5.7 puisque celle-ci assure que toute suite de Jordan-Hölder de $L$ admet un et un seul sous-quotient qui ne provient pas de $S$.

On sait déjà que $\operatorname{Rad}_{\mathbb{P}(V) / S}(K)$ est pervers d'après l'assertion (i) de la proposition 5.7. Considérons une filtration

$$
(0)=L_{0} \subset L_{1} \subset L_{2} \subset L_{3}=L=\operatorname{Rad}_{\mathbb{P}(V) / S}(K)
$$

comme dans l'assertion (i) déjà démontrée. Si $L_{1}=\left(\bar{\pi}^{\vee \circ}\right)^{*} M_{1}[r-1]$ pour un $\overline{\mathbb{Q}}_{\ell}$-faisceau pervers $M_{1}$ sur $S$, on a

$$
\operatorname{Hom}\left(L_{1}, L\right)=\operatorname{Hom}\left(M_{1}[r-1],\left(\bar{\pi}^{\vee \circ}\right)_{*} L\right)
$$

TOME $131-2003-\mathrm{N}^{\mathrm{O}} 4$ 
dans $D_{\mathrm{c}}^{\mathrm{b}}\left(S, \overline{\mathbb{Q}}_{\ell}\right)$. Par suite, on a $\operatorname{Hom}\left(L_{1}, L\right)=(0)$, et nécessairement $L_{1}=(0)$, puisque $\left(\bar{\pi}^{\vee 0}\right)_{*} L=(0)$ (cf. l'assertion (ii) de la proposition 5.4). Comme les hypothèses sur $K$ sont auto-duales, on voit de même que $L / L_{2}=(0)$, d'où le corollaire.

\section{BIBLIOGRAPHIE}

[1] Behrend (K.) - Derived $\ell$-adic categories for algebraic stacks, Mem. Amer. Math. Soc., vol. 163, American Mathematical Society, 2003.

[2] Beilinson (A.), Bernstein (J.) \& Deligne (P.) - Faisceaux pervers, Astérisque, vol. 100, Société Mathématique de France, 1982.

[3] Brylinski (J.-L.) - Transformations canoniques, dualité projective, théorie de Lefschetz, transformations de Fourier et sommes trigonométriques, in Géométrie et analyse microlocales, Astérisque, vol. 140-141, Société Mathématique de France, 1986, pp. 3-134.

[4] Ekedahl (T.) - On the Adic Formalism, in The Grothendieck Festschrift, vol. II, Progess in Mathematics, vol. 87, Birkhäuser, 1990, pp. 197-218.

[5] Frenkel (E.), Gaitsgory (D.) \& Vilonen (K.) - On the geometric Langlands conjecture, J. Amer. Math. Soc., t. 15 (2002), pp. 367-417.

[6] Gabber (O.) - Notes on some t-structures, manuscrit, décembre 2000.

[7] Gabriel (P.) - Des catégories abéliennes, Bull. Soc. Math. France, t. 90 (1962), pp. 323-448.

[8] Katz (N.M.) \& LAumon (G.) - Transformation de Fourier et majoration de sommes exponentielles, Publ. Math. IHES, t. 62 (1986), pp. 145-202.

[9] Laumon (G.) - Transformation de Fourier, constantes d'équations fonctionnelles et conjecture de Weil, Publ. Math. IHES, t. 65 (1987), pp. 131210.

[10] _ Travaux de Frenkel, Gaitsgory et Vilonen sur la correspondance de Drinfeld-Langlands, Séminaire Bourbaki, exposé no 906, 2002.

[11] Laumon (G.) \& Moret-Bailly (L.) - Champs algébriques, SpringerVerlag, 1999.

[12] SPRinger (T.) - A purity result for fixed point varieties in flag manifolds, J. Fac. Sci. Univ. Tokyo, t. 31 (1984), pp. 271-282. 